\title{
36. MINOR ELEMENT AND STABLE ISOTOPIC COMPOSITION OF THE CARBONATE FINE FRACTION: SITE 709, INDIAN OCEAN
}

\author{
Paul A. Baker, ${ }^{2}$ Mitchell J. Malone, ${ }^{2}$ Stephen J. Burns,${ }^{3}$ and Peter K. Swart ${ }^{3}$
}

\begin{abstract}
Stable isotopic and minor element compositions were measured on the fine fraction of pelagic carbonate sediments from Ocean Drilling Program Site 709 in the central Indian Ocean. This section ranges in age from 47 Ma to the present. The observed compositional variations are the result of either paleoceanographic changes (past oceanic chemical or temperature variations) or diagenetic changes.

The $\mathrm{CaCO}_{3}$ record is little affected by diagenesis. From previous work, carbonate content is known to be determined by the interplay of biological productivity, water column dissolution, and dilution. The carbon isotopic record is generally similar to previously published curves. A good correlation was observed between sea-level high stands and high ${ }^{13} \mathrm{C} /{ }^{12} \mathrm{C}$ ratios. This supports Shackleton's hypothesis that as the proportion of organic carbon buried in marine sediments becomes larger, oceanic-dissolved inorganic carbon becomes isotopically heavier. This proportion appears to be higher when sea level is higher and organic carbon is buried in more extensive shallow-shelf sediments.

The strontium content and oxygen isotopic composition of carbonate sediments are much more affected by burial diagenesis. Low strontium concentrations are invariably associated with high values of $\delta^{18} \mathrm{O}$, probably indicating zones of greater carbonate recrystallization. Nevertheless, there is an inverse correlation between strontium concentration and sea level that is thought to be a result of high-strontium aragonitic sedimentation on shallow banks and shelves during high stands.

Iron and manganese concentrations and, to a lesser extent, magnesium and strontium concentrations and carbon isotopic ratios are affected by early diagenetic reactions. These reactions are best observed in a slumped interval of sediments that occurs between 13.0 and $17.5 \mathrm{Ma}$. As a result of microbial reduction of manganese and iron oxides and dissolved sulfate, it is hypothesized that small amounts of mixed-metal carbonate cements are precipitated. These have low carbon isotopic ratios and high concentrations of metals.
\end{abstract}

\section{INTRODUCTION}

A great deal of work in recent years has been carried out on the reconstruction of Cenozoic ocean temperature and composition from the isotopic and chemical compositions of marine carbonate sediments. Most often, these studies have been based upon the measurement of stable oxygen and carbon isotopic ratios in foraminifers or total carbonate compositions of bulk sediments. Other measurements have included various elemental concentrations in the carbonates (e.g., cadmium, lithium, barium, strontium, magnesium, iron, manganese, sodium, and rare earth elements) and various isotopic ratios (e.g., neodymium and strontium). For some of these parameters, the sources of secular variation are reasonably well understood (e.g., total carbonate, and strontium-, carbon-, and oxygen-isotopic ratios) and useful interpretations can be made from measured data. For many of the measurements, however, especially for many of the minor elements, virtually ad hoc decisions have been made, partly by necessity, as to what processes control their measured variations.

In this study we present minor element and stable isotopic measurements made on a large suite of carefully cleaned samples of the fine fraction of carbonate sediments from Site 709. We propose that the major processes controlling the concentrations of all of the reported elements and isotopes are (1) ancient ocean surface temperature at the time of carbonate precipitation, (2) ancient ocean chemical composition at the time of car-

\footnotetext{
${ }^{1}$ Duncan, R. A., Backman, J., Peterson, L. C., et al., 1990. Proc. ODP, Sci. Results, 115: College Station, TX (Ocean Drilling Program).

2 Department of Geology, Duke University, Durham, NC 27708, U.S.A.

3 Rosenstiel School of Marine and Atmospheric Sciences, University of Miami, 4600 Rickenbacker Causeway, Miami, FL 33149, U.S.A.
}

bonate precipitation, and (3) near-surface or deeper burial diagenesis. All of these processes are important in some cases and, as will be seen, it is often very difficult to deduce the fractional role played by each. Each element or stable isotopic ratio responds differently to each of the forcing variables. For a variety of reasons, we think that a relative ranking of the presently reported parameters in terms of the fidelity of their recording of paleotemperature or paleochemistry (instead of diagenesis) is: $\mathrm{CaCO}_{3}>\delta^{13} \mathrm{C}>$ strontium $>$ magnesium $>$ iron $>$ manganese $>\delta^{18} \mathrm{O}$. That is, manganese concentrations in carbonates should be a lot more susceptible to diagenetic alteration than total carbonate variations. If minor elemental variations coincide with variations of total carbonate or $\delta^{13} \mathrm{C}$, then we reason that they may be reflecting true paleoceanographic variations rather than postdepositional changes.

\section{METHODS}

Samples at Site 709 were collected with the hydraulic piston corer and the advanced hydraulic piston corer. A total of approximately 220 samples were used in this study. Approximately one $\mathrm{g}$ of each sample was treated for $30 \mathrm{~min}$ with $30 \mathrm{ml}$ of $3 \%$ $\mathrm{H}_{2} \mathrm{O}_{2}$ in a water bath maintained at $55^{\circ} \mathrm{C}$. This procedure disaggregated the samples and oxidized any organic carbon present in the samples. The samples were then wet sieved with deionized water and the fine fraction $(<63 \mu \mathrm{m})$ was retained. Samples were centrifuged for $30 \mathrm{~min}$ and the supernatant liquid was decanted. A reductive cleaning step was performed next with a mixed reagent freshly prepared by combining $40 \mathrm{ml}$ of $0.3 \mathrm{M}$ sodium citrate and $5 \mathrm{ml}$ of $1.0 \mathrm{M}$ sodium bicarbonate. Samples were reacted for $30 \mathrm{~min}$ in a water bath at $55^{\circ} \mathrm{C}$ with $9 \mathrm{ml}$ of mixed reagent plus $0.5 \mathrm{~g}$ of sodium dithionate. Samples were then centrifuged and the supernatant was decanted. The samples were rinsed three times with distilled water and centrifuged after each rinse. Finally, the samples were dried and precisely 
weighed subsamples of about $50 \mathrm{mg}$ were dissolved in $50 \mathrm{ml}$ of ammonium acetate-acetic acid $(\mathrm{pH}=5.5)$ solution for about 10 $\mathrm{min}$ at room temperature. The samples were centrifuged for 30 min and the supernatant liquid was saved for analysis.

All elemental analyses were conducted with standard methods using a Perkin Elmer Model 5000 atomic absorption spectrophotometer. Replicate analyses indicate the following relative errors: $1.7 \%$ for $\mathrm{CaCO}_{3}, 2.2 \%$ for strontium, $3.4 \%$ for magnesium, $6.4 \%$ for manganese, and $24.4 \%$ for iron. Since typical instrumental errors are about $1 \%-2 \%$ for all elements, it is clear that sample heterogeneity is responsible for the large uncertainties in manganese and iron determinations. Uncertainties in $\mathrm{CaCO}_{3}$ determinations are included in all the other elemental data.

Stable isotopic analyses were performed on fine-fraction samples that had been treated in exactly the same fashion as the samples for elemental analysis (except for the final dissolution step). Carbon and oxygen isotopic analyses were conducted with phosphoric acid dissolution using an automated on-line device reacting at $90^{\circ} \mathrm{C}$ for $10 \mathrm{~min}$. Extracted $\mathrm{CO}_{2}$ was analyzed on a MAT 251 stable isotope ratio mass spectrometer at the University of Miami. All results are reported relative to the PDB standard. External error, determined by analyses of 20 replicate samples, is $\pm 0.026 \%$ for $\delta^{18} \mathrm{O}$ and $\pm 0.016 \%$ for $\delta^{13} \mathrm{C}$.

Sample ages were derived from nannofossil zonation and the ages for the zonal boundaries are presented in Shipboard Scientific Party (1988a, table 3). Within the zones, ages were determined by interpolation assuming constant sedimentation rates.

\section{RESULTS AND DISCUSSION}

All of the chemical and isotopic results from this study are presented in Table 1. The most relevant data are shown in Figures 1 through 7 .

\section{Carbonate Contents}

Carbonate contents of the sediments are shown in Figure 1. The vast majority of samples have carbonate contents greater than $80 \%$, making them suitable for this study. These data compare quite well with the carbonate determinations of bulk samples shown in Shipboard Scientific Party (1988b, p. 486, fig. 20). Major features include a carbonate low around $47 \mathrm{Ma}$, an increase in carbonate from 47 to $31 \mathrm{Ma}$, and two carbonate highs at 31 and $26 \mathrm{Ma}$ with an intervening low at $29 \mathrm{Ma}$. There is a carbonate low at $20 \mathrm{Ma}$ followed by a high centered at 18 $\mathrm{Ma}$. This is followed by an interval of slumping between approximately 13.0 and $17.5 \mathrm{Ma}$ (Shipboard Scientific Party, 1988b, p. 468); within this interval there are two discrete fluctuations of carbonate content. Each is associated with a slump block. The carbonate contents are thought to be repeats of the top of the underlying, nonslumped interval. Since $13 \mathrm{Ma}$, fluctuations have been somewhat smaller with a significant carbonate low showing up around $10 \mathrm{Ma}$ (seen much better in the data of Shipboard Scientific Party, 1988b) and a carbonate high around $5 \mathrm{Ma}$. There appears to be some correlation between the overall sedimentation rate and the carbonate content (higher sedimentation rates are associated with higher carbonate contents), but the sedimentation rates are not very well constrained at this resolution.

\section{Carbon Isotopes}

Carbon isotopic data are shown in Figure 2. This record is similar to the carbon isotopic compositions of bulk carbonate sediments of the same age at Deep Sea Drilling Project (DSDP) Sites 525 and 528 reported by Shackleton and Hall (1984) and Shackleton (1987). The first-order features of both data sets are: high, positive values of $\delta^{13} \mathrm{C}$ from 47 to about $20 \mathrm{Ma}$, low or negative values at present, and a period of decrease of $\delta^{13} \mathrm{C}$ from $10 \mathrm{Ma}$ to the present. One notable feature, different from previously published curves is the $1.6 \%$ decrease in $\delta^{13} \mathrm{C}$ in the early Miocene. Because this decrease is coincident with large increases in iron, manganese, and magnesium, however, we think that it is produced by a diagenetic process that occurs during slumping (discussed below). It should be noted that the isotopic change is also coincident with a sharp decrease in the carbonate mass accumulation rate (Peterson and Backman, this volume) at neighboring (nonslumped) sites.

It is evident that there is also fine structure in the carbon isotopic record at Site 709 . Before $20 \mathrm{Ma}$ there are about five isotopic excursions, each lasting about $5 \mathrm{~m}$.y. There are two major positive excursions in this period. These occur during the early Oligocene (about $32 \mathrm{Ma}$ ) and just after the Oligocene/Miocene boundary (about $23 \mathrm{Ma}$ ). There are three major negative excursions in the same period. They are centered at about $36 \mathrm{Ma}$ (near the Eocene/Oligocene boundary), $30 \mathrm{Ma}$, and $26 \mathrm{Ma}$.

Shackleton (1987) interpreted the decrease in the $\delta^{13} \mathrm{C}$ of bulk carbonate sediments since the middle Miocene as a global oceanic signal reflecting a long-term decrease in the amount of organic carbon buried in sediments. Similarly, the positive excursions before $20 \mathrm{Ma}$ may represent shorter time periods of globally increased burial of organic carbon. Vincent and Berger (1985) identified a positive carbon isotopic excursion of about 1 per mil lasting about 4 m.y. centered on 15.5 Ma (also observed by Shackleton, 1987). This shift was seen in both benthic and planktonic foraminifers and thus is considered to reflect a global oceanic change. Vincent and Berger (1985) suggested that this shift was produced by the increased burial of organic carbon beneath regions of high productivity. At Site 709 there is a positive isotopic excursion of about 1 per mil in about the same time interval, although because of the slumping in the 13-17.5 Ma interval at this site, the exact stratigraphic position of this isotopic peak is unknown.

One of the most obvious means of increasing the amount of carbon buried in marine sediments is to increase the area of shallow-water sedimentation by eustatic sea-level rise. Positive $\delta^{13} \mathrm{C}$ excursions, therefore, should be associated with high eustatic sea levels. This prediction seems to be borne out in applying the Cenozoic eustatic sea-level cycle chart of Haq et al. (1987) converted to the Leg 115 time scale. High sea-level stands occurred from 50 to $45 \mathrm{Ma}, 42$ to $40 \mathrm{Ma}, 35$ to $30 \mathrm{Ma}, 24$ to $23 \mathrm{Ma}$, and 17 to $14 \mathrm{Ma}$. Each of these intervals, including the excursion discussed by Vincent and Berger (1985), is also a time of higher $\delta^{13} \mathrm{C}$.

These conclusions must be weighed carefully. Miller and Fairbanks (1985) found quite different timing of "global" carbon isotopic cycles in the Oligocene and Miocene epochs, and they concluded that these cycles were independent of eustatic sealevel changes. Shackleton (1987) concluded that the possible associations between $\delta^{13} \mathrm{C}$ events and sea level could not "be tested adequately until the sea-level record is better known and understood." Shackleton (1987) did not have available to him the chronology and sea-level cyclicity chart published by Haq et al. (1987). In fact, since the Paleocene, there does seem to be coincidence between sea-level highstands (Haq et al., 1987) and Shackleton's (1987) positive $\delta^{13} \mathrm{C}$ excursions.

\section{Strontium}

Strontium concentrations of ancient marine carbonate sediments were measured previously by many workers. It has been proposed that these concentrations were influenced by several factors, including leaching of strontium from noncarbonate sources during sample preparation, variations of the oceanic ratio of dissolved strontium-to-calcium $(\mathrm{Sr} / \mathrm{Ca})$ (e.g., Graham et al., 1982; Renard, 1986), variations of oceanic temperature (Kinsman, 1969), and diagenesis (e.g., Baker et al., 1982). We think 
Table 1. Elemental and stable isotopic compositions of fine fraction samples from Site 709.

\begin{tabular}{|c|c|c|c|c|c|c|c|c|c|c|c|}
\hline Core, section & $\begin{array}{l}\text { Depth } \\
\text { (mbsf) }\end{array}$ & $\begin{array}{c}\mathrm{Wt}_{\mathrm{t}} \\
(\mathrm{mg})\end{array}$ & $\begin{array}{c}\mathrm{CaCO}_{3} \\
\text { (mg) }\end{array}$ & $\begin{array}{c}\mathrm{CaCO}_{3} \\
(\mathrm{wt} \%)\end{array}$ & $\begin{array}{c}\mathrm{Sr} \\
(\mathrm{ppm})\end{array}$ & $\begin{array}{c}\mathrm{Mn} \\
(\mathrm{ppm})\end{array}$ & $\begin{array}{c}\mathrm{Fe} \\
(\mathrm{ppm})\end{array}$ & $\begin{array}{c}\mathrm{Mg} \\
(\mathrm{ppm})\end{array}$ & $\delta^{13} \mathrm{C}$ & $\delta^{18} \mathrm{O}$ & $\begin{array}{l}\text { Age } \\
\text { (Ma) }\end{array}$ \\
\hline \multicolumn{12}{|l|}{$115-709 \mathrm{~A}-$} \\
\hline $1 \mathrm{H}-1$ & 0.98 & 49.8 & 44.77 & 89.90 & 1798 & 268 & 480 & 905 & 0.35 & -0.20 & 0.11 \\
\hline $1 \mathrm{H}-2$ & 2.48 & 50.0 & 43.77 & 87.54 & 2011 & 217 & 605 & 685 & 0.13 & -0.60 & 0.27 \\
\hline $1 \mathrm{H}-3$ & 3.98 & 50.8 & 44.77 & 88.13 & 2122 & 212 & 681 & 748 & 0.41 & -0.02 & 0.37 \\
\hline $1 \mathrm{H}-4$ & 5.48 & 50.8 & 47.27 & 93.05 & 2084 & 159 & 635 & 497 & 1.09 & -0.09 & 0.46 \\
\hline $1 \mathrm{H}-6$ & 8.48 & 50.0 & 43.39 & 86.79 & 2063 & 311 & 553 & 772 & -0.22 & -0.38 & 0.79 \\
\hline $1 \mathrm{H}-7$ & 9.88 & 50.9 & 41.02 & 80.58 & 2048 & 280 & 549 & 987 & -0.39 & -0.11 & 0.95 \\
\hline $2 \mathrm{H}-1$ & 11.08 & 49.9 & 39.64 & 79.44 & 2220 & 202 & 744 & 757 & 0.25 & -0.50 & 1.08 \\
\hline $2 \mathrm{H}-2$ & 12.58 & 49.0 & 41.39 & 84.47 & 2029 & 387 & 640 & 858 & 0.61 & -0.45 & 1.25 \\
\hline $2 \mathrm{H}-3$ & 14.08 & 50.9 & 44.14 & 86.72 & 1994 & 284 & 544 & 872 & 0.25 & -0.25 & 1.42 \\
\hline $2 \mathrm{H}-6$ & 18.58 & 49.3 & 43.02 & 87.26 & 1953 & 372 & 802 & 872 & 0.22 & -0.13 & 1.94 \\
\hline $3 \mathrm{H}-1$ & 20.68 & 49.7 & 41.64 & 83.79 & 2233 & 276 & 516 & 949 & 0.22 & -0.03 & 2.14 \\
\hline $3 \mathrm{H}-2$ & 22.18 & 50.6 & 44.14 & 87.24 & 1710 & 204 & 510 & 770 & 0.32 & 0.09 & 2.28 \\
\hline $3 \mathrm{H}-3$ & 23.68 & 50.0 & 43.77 & 87.54 & 2011 & 366 & 765 & 982 & 0.48 & -0.77 & 2.42 \\
\hline $3 \mathrm{H}-4$ & 25.18 & 50.8 & 40.64 & 80.00 & 1858 & 381 & 554 & 1316 & -0.23 & -0.32 & 2.56 \\
\hline $3 \mathrm{H}-5$ & 26.68 & 50.4 & 42.39 & 84.11 & 2076 & 295 & 401 & 1050 & 0.21 & -0.58 & 2.70 \\
\hline $3 \mathrm{H}-6$ & 28.18 & 50.5 & 43.02 & 85.18 & 2080 & 321 & 337 & 872 & 0.40 & -0.41 & 2.83 \\
\hline $4 \mathrm{H}-1$ & 30.38 & 50.0 & 30.63 & 61.28 & 2742 & 457 & 212 & 1290 & 0.28 & -0.58 & 3.04 \\
\hline $4 \mathrm{H}-2$ & 31.88 & 50.0 & 32.64 & 65.28 & 2803 & 444 & 276 & 1103 & 0.31 & -0.53 & 3.18 \\
\hline $4 \mathrm{H}-3$ & 33.38 & 51.0 & 33.01 & 64.73 & 2545 & 515 & 197 & 1091 & 0.34 & -0.94 & 3.32 \\
\hline $4 \mathrm{H}-4$ & 34.88 & 49.8 & 42.77 & 83.86 & 1847 & 444 & 281 & 982 & 0.38 & -0.29 & 3.46 \\
\hline $4 \mathrm{H}-5$ & 35.8 & 49.9 & 41.02 & 82.20 & 1792 & 414 & 219 & 1110 & -0.16 & -0.50 & 3.54 \\
\hline $6 \mathrm{H}-1$ & 49.68 & 50.0 & 44.14 & 88.29 & 1869 & 261 & 295 & 997 & 0.64 & 0.19 & 4.40 \\
\hline $6 \mathrm{H}-1$ & 49.68 & 50.1 & 43.77 & 87.36 & 1919 & 274 & 331 & 1017 & 0.64 & 0.19 & 4.40 \\
\hline $6 \mathrm{H}-1$ & 49.68 & 49.3 & 45.14 & 91.27 & 1783 & 255 & 354 & 964 & 0.64 & 0.19 & 4.40 \\
\hline $6 \mathrm{H}-1$ & 49.68 & 50.3 & 45.52 & 90.49 & 1812 & 253 & 286 & 956 & 0.64 & 0.19 & 4.40 \\
\hline $6 \mathrm{H}-2$ & 51.18 & 50.7 & 45.89 & 90.52 & 1830 & 240 & 490 & 904 & 0.00 & 0.04 & 4.49 \\
\hline $6 \mathrm{H}-3$ & 52.68 & 50.0 & 45.52 & 91.04 & 1845 & 242 & 319 & 923 & 0.54 & 0.17 & 4.58 \\
\hline $6 \mathrm{H}-4$ & 54.18 & 50.5 & 45.52 & 90.14 & 1692 & 297 & 406 & 1033 & 1.04 & 0.59 & 4.66 \\
\hline $6 \mathrm{H}-5$ & 55.68 & 51.0 & 45.14 & 88.52 & 1706 & 244 & 476 & 1008 & 0.21 & -0.33 & 4.73 \\
\hline $6 \mathrm{H}-6$ & 57.18 & 50.7 & 45.14 & 89.04 & 1750 & 277 & 476 & 997 & 0.56 & 0.07 & 4.81 \\
\hline $7 \mathrm{H}-1$ & 59.28 & 49.7 & 43.77 & 88.06 & 1759 & 308 & 605 & 1040 & 0.82 & 0.27 & 4.91 \\
\hline $7 \mathrm{H}-2$ & 60.78 & 50.7 & 43.77 & 86.33 & 1805 & 286 & 583 & 1040 & -0.01 & -0.21 & 4.99 \\
\hline $7 \mathrm{H}-3$ & 62.28 & 50.9 & 44.77 & 87.95 & 1843 & 265 & 447 & 994 & 0.35 & -0.15 & 5.17 \\
\hline $7 \mathrm{H}-4$ & 63.78 & 49.9 & 44.77 & 89.72 & 1843 & 279 & 380 & 905 & 0.56 & 0.04 & 5.14 \\
\hline $7 \mathrm{H}-5$ & 65.28 & 49.5 & 42.02 & 84.88 & 1880 & 297 & 345 & 952 & 0.81 & 0.15 & 5.22 \\
\hline $7 \mathrm{H}-6$ & 66.78 & 49.9 & 42.02 & 84.20 & 1999 & 286 & 535 & 940 & -0.05 & -0.40 & 5.30 \\
\hline $8 \mathrm{H}-1$ & 68.98 & 51.0 & 45.14 & 88.52 & 1861 & 266 & 354 & 853 & 0.80 & 0.31 & 5.41 \\
\hline $8 \mathrm{H}-2$ & 70.48 & 49.0 & 43.02 & 87.79 & 1953 & 291 & 395 & 872 & 0.62 & -0.39 & 5.49 \\
\hline $8 \mathrm{H}-3$ & 71.98 & 50.6 & 46.14 & 91.19 & 1669 & 314 & 553 & 1084 & 0.66 & 0.69 & 5.56 \\
\hline $8 \mathrm{H}-4$ & 73.48 & 50.5 & 44.14 & 87.41 & 1869 & 272 & 510 & 997 & 0.30 & 0.03 & 5.64 \\
\hline $8 \mathrm{H}-5$ & 74.98 & 51.0 & 46.14 & 90.48 & 1550 & 271 & 347 & 1062 & 1.01 & 0.16 & 5.72 \\
\hline $8 \mathrm{H}-6$ & 75.48 & 50.0 & 43.02 & 86.03 & 1755 & 209 & 523 & 930 & 0.54 & -0.30 & 5.74 \\
\hline $9 \mathrm{H}-1$ & 78.58 & 49.2 & 43.77 & 88.96 & 1634 & 286 & 388 & 1112 & 0.28 & 0.28 & 5.90 \\
\hline $9 \mathrm{H}-2$ & 80.08 & 50.9 & 42.77 & 84.02 & 1847 & 257 & 304 & 1040 & 0.61 & 0.23 & 5.98 \\
\hline $9 \mathrm{H}-3$ & 81.58 & 51.0 & 44.14 & 86.55 & 1824 & 227 & 272 & 952 & 1.04 & 0.20 & 6.06 \\
\hline $9 \mathrm{H}-4$ & 83.08 & 50.9 & 45.14 & 88.69 & 1673 & 244 & 410 & 1019 & 0.65 & 0.24 & 6.13 \\
\hline $9 \mathrm{H}-5$ & 84.58 & 50.0 & 43.02 & 86.03 & 1999 & 174 & 395 & 860 & 0.58 & -0.30 & 6.21 \\
\hline $9 \mathrm{H}-6$ & 86.08 & 49.8 & 44.52 & 89.39 & 1696 & 225 & 573 & 1033 & 0.50 & 0.00 & 6.29 \\
\hline $10 \mathrm{H}-1$ & 88.28 & 50.9 & 42.02 & 82.55 & 1880 & 274 & 607 & 1047 & 0.92 & -0.03 & 6.40 \\
\hline $10 \mathrm{H}-2$ & 89.78 & 50.0 & 38.27 & 76.53 & 2012 & 284 & 444 & 1084 & 0.78 & -0.24 & 6.48 \\
\hline $10 \mathrm{H}-3$ & 91.28 & 50.2 & 42.02 & 83.70 & 1797 & 238 & 476 & 1095 & 0.93 & 0.03 & 6.61 \\
\hline $10 \mathrm{H}-4$ & 92.78 & 51.0 & 41.39 & 81.16 & 1776 & 302 & 290 & 1148 & 0.81 & -0.44 & 6.77 \\
\hline $10 \mathrm{H}-6$ & 95.78 & 49.9 & 42.02 & 84.20 & 1916 & 238 & 309 & 976 & 0.99 & -0.81 & 7.08 \\
\hline $10 \mathrm{H}-7$ & 96.9 & 50.8 & 44.52 & 87.63 & 1606 & 303 & 382 & 1123 & 0.77 & 0.16 & 7.20 \\
\hline $11 \mathrm{H}-1$ & 97.88 & 50.7 & 45.89 & 90.52 & 1722 & 240 & 436 & 654 & 1.09 & 0.00 & 7.30 \\
\hline $11 \mathrm{H}-2$ & 99.38 & 49.8 & 44.14 & 88.64 & 1620 & 249 & 419 & 1053 & 1.76 & 0.02 & 7.46 \\
\hline $11 \mathrm{H}-3$ & 100.88 & 50.4 & 43.77 & 86.84 & 1599 & 228 & 514 & 1142 & 0.74 & -0.24 & 7.61 \\
\hline $11 \mathrm{H}-4$ & 102.38 & 50.0 & 42.77 & 85.54 & 1672 & 234 & 526 & 1076 & 0.86 & 0.00 & 7.77 \\
\hline $11 \mathrm{H}-5$ & 103.88 & 49.9 & 42.02 & 84.20 & 1749 & 262 & 345 & 1047 & 1.18 & -0.09 & 7.93 \\
\hline $11 \mathrm{H}-6$ & 105.38 & 49.9 & 41.64 & 83.45 & 2113 & 324 & 516 & 672 & 0.99 & 0.04 & 8.08 \\
\hline $12 \mathrm{H}-1$ & 107.58 & 50.3 & 43.77 & 87.01 & 1634 & 354 & 297 & 1051 & 0.81 & 0.06 & 8.31 \\
\hline $12 \mathrm{H}-2$ & 109.08 & 50.7 & 43.39 & 85.59 & 1613 & 415 & 242 & 1060 & 1.02 & -0.05 & 8.47 \\
\hline $12 \mathrm{H}-4$ & 112.08 & 50.5 & 42.77 & 84.69 & 1847 & 468 & 245 & 947 & 0.97 & -0.10 & 8.77 \\
\hline $12 \mathrm{H}-5$ & 113.58 & 50.8 & 41.39 & 81.48 & 1691 & 664 & 314 & 1256 & 0.99 & -0.09 & 9.08 \\
\hline $12 \mathrm{H}-6$ & 115.08 & 50.3 & 42.77 & 85.02 & 1882 & 736 & 281 & 1052 & 0.99 & 0.15 & 10.06 \\
\hline $13 \mathrm{H}-1$ & 117.18 & 49.8 & 42.01 & 84.37 & 1881 & 559 & 250 & 964 & 0.90 & -0.23 & 10.93 \\
\hline $13 \mathrm{H}-2$ & 118.68 & 49.2 & 41.39 & 84.13 & 1945 & 568 & 193 & 930 & 1.41 & 0.01 & 11.11 \\
\hline $13 \mathrm{H}-3$ & 120.18 & 50.5 & 46.52 & 92.12 & 1773 & 645 & 226 & 946 & 1.29 & 0.47 & 11.28 \\
\hline $13 \mathrm{H}-4$ & 121.68 & 49.3 & 42.39 & 85.99 & 1651 & 720 & 283 & 1026 & 0.95 & 0.47 & 11.45 \\
\hline $13 \mathrm{H}-5$ & 123.18 & 50.5 & 45.14 & 89.39 & 1584 & 565 & 177 & 1063 & 0.93 & 0.61 & 11.62 \\
\hline $13 \mathrm{H}-6$ & 124.68 & 50.8 & 45.52 & 89.60 & 1768 & 593 & 351 & 1142 & 0.94 & 0.45 & 11.79 \\
\hline $14 \mathrm{H}-1$ & 126.78 & 50.5 & 44.14 & 87.41 & 1586 & 510 & 419 & 1155 & 1.56 & 0.19 & 12.03 \\
\hline $14 \mathrm{H}-2$ & 128.28 & 50.0 & 42.39 & 84.78 & 1486 & 330 & 342 & 1345 & 1.30 & 0.33 & 12.20 \\
\hline $14 \mathrm{H}-3$ & 129.78 & 49.0 & 42.39 & 86.51 & 1522 & 271 & 401 & 1321 & 1.62 & 0.23 & 12.37 \\
\hline $14 \mathrm{H}-4$ & 131.28 & 50.0 & 42.39 & 84.78 & 1522 & 201 & 377 & 1321 & 0.77 & 0.13 & 12.55 \\
\hline $14 \mathrm{H}-5$ & 132.78 & 50.0 & 43.39 & 86.79 & 1567 & 196 & 369 & 1129 & 0.54 & -0.04 & 12.72 \\
\hline
\end{tabular}


Table 1 (continued).

\begin{tabular}{|c|c|c|c|c|c|c|c|c|c|c|c|}
\hline Core, section & $\begin{array}{l}\text { Depth } \\
\text { (mbsf) }\end{array}$ & $\begin{array}{c}\mathrm{Wt} \\
(\mathrm{mg})\end{array}$ & $\underset{(\mathrm{mg})}{\mathrm{CaCO}_{3}}$ & $\begin{array}{l}\mathrm{CaCO}_{3} \\
(\mathrm{wt} \%)\end{array}$ & $\begin{array}{c}\mathrm{Sr} \\
(\mathrm{ppm})\end{array}$ & $\underset{(\mathrm{ppm})}{\mathrm{Mn}}$ & $\begin{array}{c}\mathrm{Fe} \\
(\mathrm{ppm})\end{array}$ & $\underset{(\mathrm{ppm})}{\mathrm{Mg}}$ & $\delta^{13} \mathrm{C}$ & $\delta^{18} \mathrm{O}$ & $\begin{array}{l}\text { Age } \\
\text { (Ma) }\end{array}$ \\
\hline \multicolumn{12}{|l|}{ 115-709A- (Cont.) } \\
\hline $14 \mathrm{H}-6$ & 134.28 & 50.0 & 40.89 & 81.78 & 1492 & 220 & 257 & 1296 & 0.55 & 0.04 & 12.89 \\
\hline $15 \mathrm{H}-1$ & 136.48 & 49.8 & 43.02 & 86.38 & 1709 & 232 & 337 & 1069 & 0.55 & 0.33 & 13.14 \\
\hline $15 \mathrm{H}-2$ & 137.98 & 50.0 & 43.02 & 86.03 & 1709 & 291 & 279 & 976 & 0.37 & -0.13 & 13.36 \\
\hline $15 \mathrm{H}-3$ & 139.48 & 49.2 & 43.39 & 88.20 & 1613 & 207 & 150 & 1037 & 0.64 & -0.01 & 13.61 \\
\hline $15 \mathrm{H}-4$ & 140.98 & 50.8 & 44.14 & 86.90 & 1620 & 861 & 272 & 1405 & 1.48 & -0.07 & 13.85 \\
\hline $15 \mathrm{H}-5$ & 142.48 & 50.0 & 44.52 & 89.04 & 1527 & 752 & 382 & 1269 & 1.56 & -0.02 & 14.10 \\
\hline $15 \mathrm{H}-6$ & 143.98 & 49.9 & 44.52 & 89.21 & 1651 & 472 & 292 & 1269 & 1.34 & -0.03 & 14.34 \\
\hline $16 \mathrm{H}-1$ & 146.18 & 49.6 & 41.39 & 83.45 & 1691 & 532 & 447 & 1474 & 1.20 & -0.18 & 14.70 \\
\hline $16 \mathrm{H}-2$ & 147.68 & 49.8 & 45.14 & 90.65 & 1506 & 521 & 532 & 1174 & 0.90 & -0.36 & 14.95 \\
\hline $16 \mathrm{H}-3$ & 149.18 & 49.9 & 43.02 & 86.21 & 1709 & 511 & 395 & 1104 & 0.94 & -0.24 & 15.19 \\
\hline $16 \mathrm{H}-4$ & 150.68 & 50.0 & 44.77 & 89.54 & 1485 & 313 & 380 & 1195 & 0.76 & -0.03 & 15.44 \\
\hline $16 \mathrm{H}-5$ & 152.18 & 50.5 & 43.39 & 85.93 & 1613 & 346 & 392 & 1210 & 0.45 & 0.08 & 15.68 \\
\hline $16 \mathrm{H}-6$ & 153.68 & 50.0 & 43.02 & 86.03 & 1627 & 349 & 395 & 1186 & 0.49 & -0.04 & 15.93 \\
\hline $17 \mathrm{H}-1$ & 155.83 & 49.9 & 36.14 & 72.42 & 2089 & 789 & 664 & 1314 & 1.32 & 0.03 & 16.28 \\
\hline $17 \mathrm{H}-2$ & 157.33 & 50.0 & 38.89 & 77.78 & 1890 & 669 & 437 & 1234 & 1.01 & -0.16 & 16.53 \\
\hline $17 \mathrm{H}-3$ & 158.83 & 50.2 & 39.64 & 78.97 & 1678 & 568 & 505 & 1337 & 0.75 & 0.42 & 16.77 \\
\hline $17 \mathrm{H}-4$ & 160.33 & 50.1 & 44.14 & 88.11 & 1461 & 498 & 419 & 1201 & 0.53 & 0.15 & 17.02 \\
\hline $17 \mathrm{H}-5$ & 161.83 & 50.0 & 43.39 & 86.79 & 1567 & 507 & 334 & 1152 & 0.66 & 0.13 & 17.26 \\
\hline $17 \mathrm{H}-6$ & 163.33 & 50.9 & 44.52 & 87.46 & 1887 & 494 & 505 & 977 & 1.05 & -0.06 & 17.51 \\
\hline $18 \mathrm{H}-1$ & 165.58 & 51.0 & 44.77 & 87.78 & 1686 & 469 & 447 & 1061 & 0.67 & 0.06 & 17.88 \\
\hline $18 \mathrm{H}-2$ & 167.08 & 49.1 & 43.39 & 88.38 & 1740 & 323 & 300 & 1221 & 0.56 & 0.50 & 18.12 \\
\hline $18 \mathrm{H}-3$ & 168.58 & 50.1 & 43.39 & 86.61 & 1775 & 876 & 461 & 968 & 0.36 & -0.06 & 18.37 \\
\hline $18 \mathrm{H}-4$ & 170.08 & 49.1 & 42.02 & 85.57 & 1797 & 940 & 190 & 1000 & 0.59 & 0.04 & 18.62 \\
\hline $18 \mathrm{H}-5$ & 171.58 & 49.0 & 39.89 & 81.41 & 1617 & 840 & 88 & 1203 & 0.52 & 0.19 & 19.02 \\
\hline $18 \mathrm{H}-6$ & 173.08 & 49.9 & 34.89 & 69.92 & 2049 & 946 & 530 & 1476 & 0.79 & 0.10 & 19.41 \\
\hline $19 \mathrm{H}-1$ & 175.28 & 49.6 & 38.27 & 77.15 & 1868 & 614 & 562 & 1228 & 0.84 & 0.04 & 19.99 \\
\hline $19 \mathrm{H}-2$ & 176.78 & 49.8 & 38.89 & 78.09 & 1980 & 476 & 437 & 1119 & 0.91 & 0.08 & 20.38 \\
\hline $19 \mathrm{H}-3$ & 178.28 & 50.0 & 39.64 & 79.28 & 2081 & 555 & 227 & 971 & 1.19 & -0.15 & 20.78 \\
\hline $19 \mathrm{H}-4$ & 179.6 & 50.8 & 37.52 & 73.85 & 2146 & 453 & 27 & 986 & 1.22 & -0.20 & 21.12 \\
\hline $20 \mathrm{H}-1$ & 184.88 & 50.4 & 45.52 & 90.31 & 2010 & 406 & 176 & 780 & 1.95 & 0.05 & 22.51 \\
\hline $20 \mathrm{H}-2$ & 186.38 & 49.8 & 44.14 & 88.64 & 1790 & 340 & 113 & 895 & 1.82 & 0.19 & 22.91 \\
\hline $20 \mathrm{H}-3$ & 187.88 & 49.9 & 43.77 & 87.71 & 1805 & 366 & 114 & 971 & 1.68 & 0.15 & 23.26 \\
\hline $20 \mathrm{H}-4$ & 189.38 & 50.5 & 46.52 & 92.12 & 1849 & 376 & 140 & 849 & 2.07 & -0.02 & 23.50 \\
\hline $20 \mathrm{H}-5$ & 190.88 & 49.3 & 43.39 & 88.02 & 1694 & 403 & 150 & 1060 & 1.78 & 0.30 & 23.74 \\
\hline $20 \mathrm{H}-6$ & 192.38 & 49.7 & 44.52 & 89.57 & 1853 & 382 & 180 & 921 & 1.80 & 0.23 & 23.98 \\
\hline $21 \mathrm{H}-1$ & 194.48 & 49.5 & 44.14 & 89.18 & 1790 & 306 & 113 & 1008 & 1.31 & 0.05 & 24.32 \\
\hline $21 \mathrm{H}-2$ & 195.98 & 50.0 & 46.89 & 93.79 & 1951 & 266 & 139 & 864 & 2.31 & 0.28 & 24.56 \\
\hline $21 \mathrm{H}-3$ & 197.48 & 49.8 & 44.52 & 89.39 & 1774 & 247 & 236 & 1000 & 1.31 & 0.02 & 24.80 \\
\hline $21 \mathrm{H}-4$ & 198.98 & 50.3 & 45.52 & 90.49 & 1845 & 253 & 55 & 945 & 1.08 & 0.10 & 25.04 \\
\hline $21 \mathrm{H}-5$ & 200.48 & 49.8 & 46.52 & 93.41 & 1892 & 215 & 140 & 881 & 1.18 & -0.16 & 25.28 \\
\hline $21 \mathrm{H}-5$ & 200.48 & 50.1 & 46.52 & 92.85 & 1924 & 215 & 140 & 915 & 1.18 & -0.16 & 25.28 \\
\hline $21 \mathrm{H}-5$ & 200.48 & 50.0 & 45.89 & 91.79 & 1918 & 196 & 142 & 904 & 1.18 & -0.16 & 25.28 \\
\hline $21 \mathrm{H}-5$ & 200.48 & 49.6 & 46.89 & 94.54 & 1877 & 192 & 75 & 863 & 1.18 & -0.16 & 25.28 \\
\hline $21 \mathrm{H}-6$ & 201.98 & 49.7 & 44.77 & 90.08 & 1966 & 201 & 235 & 871 & 1.34 & -0.08 & 25.54 \\
\hline \multicolumn{12}{|l|}{$115-709 \mathrm{~B}-$} \\
\hline $21 X-1$ & 188.18 & 50.9 & 46.14 & 90.66 & 1821 & 271 & 282 & 964 & 1.86 & 0.36 & 23.31 \\
\hline $21 \mathrm{X}-2$ & 189.68 & 49.3 & 44.14 & 89.54 & 1744 & 396 & 272 & 974 & 1.87 & 0.14 & 23.55 \\
\hline $21 X-3$ & 191.18 & 50.2 & 44.77 & 89.18 & 1966 & 357 & 447 & 905 & 2.52 & 0.17 & 23.79 \\
\hline $21 X-4$ & 192.68 & 49.2 & 43.02 & 87.43 & 1790 & 232 & 116 & 988 & 1.75 & 0.50 & 24.03 \\
\hline $21 X-5$ & 194.18 & 50.5 & 44.77 & 88.65 & 1876 & 391 & 145 & 927 & 1.76 & 0.17 & 24.27 \\
\hline $21 X-6$ & 195.68 & 50.0 & 46.89 & 93.79 & 1685 & 299 & 171 & 1034 & 1.69 & 0.66 & 24.51 \\
\hline $22 \mathrm{X}-1$ & 197.78 & 51.0 & 47.27 & 92.68 & 1862 & 243 & 106 & 899 & 2.03 & 0.31 & 24.84 \\
\hline $22 \mathrm{X}-2$ & 199.28 & 49.9 & 44.14 & 88.46 & 1869 & 283 & 113 & 940 & 1.56 & 0.05 & 25.08 \\
\hline $22 \mathrm{X}-3$ & 200.78 & 50.7 & 46.89 & 92.49 & 1759 & 192 & 277 & 981 & 1.26 & -0.01 & 25.33 \\
\hline $22 X-4$ & 202.28 & 50.8 & 46.52 & 91.57 & 1924 & 247 & 226 & 946 & 1.24 & 0.08 & 25.59 \\
\hline $22 X-5$ & 203.78 & 50.9 & 45.52 & 89.43 & 1966 & 242 & 77 & 923 & 1.26 & 0.00 & 25.85 \\
\hline $22 X-6$ & 205.28 & 50.4 & 45.89 & 91.06 & 1918 & 305 & 283 & 981 & 1.24 & -0.14 & 26.11 \\
\hline $23 \mathrm{X}-1$ & 207.48 & 50.0 & 47.89 & 95.79 & 1942 & 115 & 52 & 793 & 1.20 & -0.04 & 26.48 \\
\hline $23 \mathrm{X}-2$ & 208.98 & 50.9 & 45.51 & 89.43 & 1846 & 297 & 264 & 989 & 1.23 & -0.15 & 26.74 \\
\hline $23 \mathrm{X}-3$ & 210.48 & 50.7 & 44.14 & 87.07 & 1948 & 261 & 147 & 986 & 1.32 & 0.04 & 27.00 \\
\hline $23 X-3$ & 210.48 & 50.0 & 42.39 & 84.78 & 2029 & 271 & 307 & 1215 & 1.32 & 0.04 & 27.00 \\
\hline $23 \mathrm{X}-3$ & 210.48 & 50.5 & 42.02 & 83.20 & 2047 & 274 & 155 & 952 & 1.32 & 0.04 & 27.00 \\
\hline $23 X-3$ & 210.48 & 50.9 & 41.64 & 81.81 & 2113 & 300 & 192 & 997 & 1.32 & 0.04 & 27.00 \\
\hline $23 X-4$ & 211.98 & 49.8 & 41.02 & 82.36 & 2097 & 305 & 122 & 1109 & 1.19 & -0.06 & 27.25 \\
\hline $23 X-5$ & 213.48 & 50.8 & 42.02 & 82.71 & 2178 & 297 & 155 & 964 & 1.28 & -0.04 & 27.51 \\
\hline $24 X-1$ & 217.08 & 50.0 & 40.27 & 80.53 & 2222 & 372 & 323 & 981 & 1.55 & 0.10 & 28.13 \\
\hline $24 X-2$ & 218.58 & 49.9 & 39.64 & 79.44 & 2258 & 290 & 265 & 933 & 1.44 & 0.20 & 28.31 \\
\hline $24 X-3$ & 220.08 & 50.0 & 41.02 & 82.03 & 2011 & 268 & 122 & 865 & 1.61 & 0.13 & 28.47 \\
\hline $24 X-4$ & 221.58 & 50.9 & 44.77 & 87.95 & 2044 & 313 & 290 & 860 & 1.41 & -0.06 & 28.63 \\
\hline $24 X-5$ & 223.08 & 50.2 & 43.39 & 86.44 & 1936 & 288 & 150 & 922 & 1.21 & 0.07 & 28.79 \\
\hline $24 X-6$ & 224.58 & 49.8 & 41.64 & 83.62 & 2017 & 336 & 312 & 877 & 1.13 & -0.09 & 28.95 \\
\hline $25 \mathrm{X}-1$ & 226.78 & 50.7 & 44.14 & 87.07 & 2186 & 306 & 272 & 770 & 1.50 & -0.17 & 29.18 \\
\hline $25 \mathrm{X}-2$ & 228.28 & 50.2 & 43.39 & 86.44 & 1982 & 323 & 277 & 853 & 1.30 & -0.17 & 29.33 \\
\hline $25 \mathrm{X}-3$ & 229.78 & 50.9 & 44.77 & 87.95 & 1966 & 335 & 78 & 771 & 1.01 & -0.26 & 29.49 \\
\hline
\end{tabular}


Table 1 (continued).

\begin{tabular}{|c|c|c|c|c|c|c|c|c|c|c|c|}
\hline Core, section & $\begin{array}{l}\text { Depth } \\
\text { (mbsf) }\end{array}$ & $\begin{array}{l}\mathrm{Wt} \\
(\mathrm{mg})\end{array}$ & $\begin{array}{c}\mathrm{CaCO}_{3} \\
\text { (mg) }\end{array}$ & $\begin{array}{c}\mathrm{CaCO}_{3} \\
(\mathrm{wt} \%)\end{array}$ & $\begin{array}{c}\mathrm{Sr} \\
(\mathrm{ppm})\end{array}$ & $\begin{array}{c}\mathrm{Mn} \\
(\mathrm{ppm})\end{array}$ & $\begin{array}{c}\mathrm{Fe} \\
(\mathrm{ppm})\end{array}$ & $\begin{array}{c}\mathrm{Mg} \\
(\mathrm{ppm})\end{array}$ & $\delta^{13} \mathrm{C}$ & $\delta^{18} \mathrm{O}$ & $\begin{array}{l}\text { Age } \\
(\mathrm{Ma})\end{array}$ \\
\hline \multicolumn{12}{|l|}{ 115-709B-(Cont.) } \\
\hline $25 \times-4$ & 231.28 & 50.0 & 44.77 & 89.54 & 1999 & 279 & 268 & 804 & 1.26 & -0.13 & 29.65 \\
\hline $25 X-5$ & 232.78 & 51.0 & 45.89 & 89.99 & 2146 & 327 & 109 & 752 & 1.60 & -0.29 & 29.81 \\
\hline $25 \mathrm{X}-6$ & 234.28 & 50.4 & 43.77 & 86.84 & 1885 & 343 & 183 & 891 & 1.11 & -0.16 & 29.97 \\
\hline $26 \mathrm{X}-1$ & 236.48 & 50.4 & 44.52 & 88.33 & 1887 & 248 & 146 & 854 & 1.40 & -0.06 & 30.20 \\
\hline $26 X-2$ & 237.98 & 49.9 & 45.52 & 91.22 & 1615 & 253 & 176 & 923 & 0.86 & 0.22 & 30.37 \\
\hline $26 \times-3$ & 239.48 & 50.0 & 46.14 & 92.29 & 1669 & 271 & 141 & 878 & 1.21 & 0.06 & 30.54 \\
\hline $26 X-4$ & 240.98 & 51.0 & 46.89 & 91.95 & 1791 & 245 & 224 & 832 & 1.38 & 0.38 & 30.70 \\
\hline $26 X-4$ & 240.98 & 49.9 & 45.89 & 91.97 & 1754 & 272 & 261 & 795 & 1.38 & 0.38 & 30.70 \\
\hline $26 \times-4$ & 240.98 & 50.6 & 47.27 & 93.42 & 1745 & 264 & 190 & 772 & 1.38 & 0.38 & 30.70 \\
\hline $26 X-4$ & 240.98 & 50.2 & 47.89 & 95.41 & 1681 & 240 & 251 & 773 & 1.38 & 0.38 & 30.70 \\
\hline $27 X-1$ & 246.08 & 50.8 & 46.14 & 90.83 & 2059 & 142 & 76 & 715 & 1.41 & -0.05 & 31.28 \\
\hline $27 X-2$ & 247.58 & 49.5 & 44.77 & 90.44 & 1999 & 190 & 56 & 715 & 1.81 & -0.11 & 31.45 \\
\hline $27 \times-3$ & 249.08 & 50.7 & 45.14 & 89.04 & 2027 & 188 & 266 & 764 & 1.89 & 0.23 & 31.62 \\
\hline $27 \times-4$ & 250.58 & 50.0 & 44.77 & 89.54 & 1921 & 123 & 78 & 804 & 1.53 & 0.01 & 31.78 \\
\hline $27 X-5$ & 252.08 & 50.0 & 43.77 & 87.54 & 1919 & 206 & 297 & 845 & 1.96 & -0.02 & 31.95 \\
\hline
\end{tabular}

$115-709 \mathrm{C}$ -

\begin{tabular}{|c|c|c|c|c|c|c|c|c|c|c|c|}
\hline $26 \times-1$ & 238.38 & 49.9 & 44.14 & 88.46 & 1790 & 283 & 147 & 816 & 1.18 & 0.09 & 30.41 \\
\hline $26 \mathrm{X}-2$ & 239.88 & 49.9 & 45.89 & 91.97 & 1798 & 251 & 54 & 774 & 1.33 & 0.01 & 30.58 \\
\hline $26 \mathrm{X}-3$ & 241.38 & 50.6 & 46.14 & 91.19 & 1821 & 217 & 76 & 726 & 1.49 & 0.04 & 30.75 \\
\hline $26 \times-4$ & 242.88 & 50.6 & 44.77 & 88.47 & 1720 & 246 & 112 & 648 & 1.71 & 0.55 & 30.92 \\
\hline $26 X-5$ & 244.38 & 50.5 & 47.89 & 94.84 & 2099 & 209 & 167 & 606 & 1.91 & -0.13 & 31.09 \\
\hline $26 X-6$ & 245.88 & 50.3 & 46.14 & 91.74 & 1983 & 195 & 173 & 726 & 1.67 & -0.25 & 31.26 \\
\hline $27 X-1$ & 247.98 & 50.7 & 44.14 & 87.07 & 2152 & 227 & 113 & 759 & 1.87 & 0.05 & 31.49 \\
\hline $27 X-2$ & 249.48 & 50.1 & 43.77 & 87.36 & 1919 & 195 & 240 & 777 & 1.80 & 0.07 & 31.66 \\
\hline $27 X-3$ & 250.98 & 50.3 & 43.02 & 85.52 & 1953 & 256 & 116 & 802 & 1.85 & 0.01 & 31.83 \\
\hline $28 \mathrm{X}-1$ & 257.68 & 50.3 & 42.02 & 83.53 & 1880 & 381 & 190 & 1069 & 1.14 & 0.05 & 32.58 \\
\hline $28 X-2$ & 259.18 & 50.6 & 46.14 & 91.19 & 1712 & 368 & 141 & 899 & 1.33 & 0.12 & 32.75 \\
\hline $28 X-3$ & 260.68 & 49.9 & 44.77 & 89.72 & 1921 & 223 & 145 & 838 & 1.43 & -0.12 & 32.92 \\
\hline $29 X-1$ & 267.38 & 49.9 & 44.77 & 89.72 & 1876 & 335 & 112 & 893 & 1.28 & -0.13 & 33.67 \\
\hline $29 X-2$ & 268.88 & 50.2 & 45.14 & 89.93 & 1949 & 310 & 144 & 820 & 1.36 & -0.25 & 33.86 \\
\hline $29 X-3$ & 270.38 & 49.8 & 34.89 & 70.06 & 2107 & 502 & 72 & 1290 & 1.20 & 0.00 & 34.08 \\
\hline $29 \times-4$ & 271.88 & 50.0 & 38.27 & 76.53 & 1973 & 327 & 170 & 1202 & 1.22 & 0.12 & 34.30 \\
\hline $29 X-5$ & 273.88 & 50.6 & 38.27 & 76.53 & 1921 & 327 & 131 & 1320 & 1.30 & 0.25 & 34.59 \\
\hline $29 X-6$ & 274.88 & 50.0 & 36.51 & 73.03 & 1863 & 370 & 68 & 1452 & 1.53 & 0.58 & 34.74 \\
\hline $30 X-1$ & 277.08 & 50.2 & 43.77 & 87.19 & 2090 & 286 & 57 & 674 & 1.26 & -0.75 & 35.62 \\
\hline $30 X-1$ & 277.08 & 50.4 & 43.39 & 86.10 & 2063 & 288 & 58 & 807 & 1.26 & -0.75 & 35.62 \\
\hline $30 X-1$ & 277.08 & 49.8 & 43.02 & 86.88 & 2080 & 267 & 116 & 732 & 1.26 & -0.75 & 35.62 \\
\hline $30 X-1$ & 277.08 & 50.0 & 43.02 & 86.03 & 2080 & 290 & 151 & 732 & 1.26 & -0.75 & 35.62 \\
\hline $30 \times-2$ & 278.58 & 50.0 & 44.14 & 88.29 & 1461 & 249 & 204 & 1291 & 1.61 & 0.50 & 36.07 \\
\hline $30 \times-3$ & 280.08 & 50.8 & 45.14 & 88.86 & 1750 & 244 & 111 & 1086 & 2.10 & 0.46 & 36.31 \\
\hline $30 \times-4$ & 281.58 & 50.0 & 44.76 & 89.54 & 2000 & 257 & 179 & 385 & 1.28 & -0.79 & 36.55 \\
\hline $30 \times-5$ & 283.08 & 50.5 & 44.76 & 88.65 & 1486 & 302 & 235 & 530 & 1.21 & -0.26 & 36.77 \\
\hline $31 X-1$ & 286.78 & 50.5 & 43.76 & 87.54 & 1348 & 263 & 80 & 1337 & 1.51 & 0.09 & 37.20 \\
\hline $31 \times-2$ & 288.28 & 50.0 & 45.14 & 90.29 & 1152 & 255 & 55 & 1495 & 1.89 & 0.28 & 37.38 \\
\hline $31 X-3$ & 289.78 & 50.1 & 43.76 & 87.36 & 1348 & 251 & 240 & 1337 & 1.89 & 0.18 & 37.55 \\
\hline $31 X-4$ & 291.28 & 50.3 & 44.77 & 89.00 & 1318 & 279 & 145 & 1340 & 1.78 & -0.04 & 37.73 \\
\hline $31 X-5$ & 292.78 & 50.5 & 40.64 & 80.48 & 1550 & 283 & 123 & 1156 & 1.63 & -0.50 & 37.90 \\
\hline $32 X-1$ & 296.38 & 50.0 & 41.64 & 83.28 & 1465 & 204 & 216 & 1189 & 1.66 & -0.03 & 38.32 \\
\hline $32 X-2$ & 297.88 & 50.6 & 44.14 & 88.00 & 1257 & 193 & 113 & 1201 & 1.69 & 0.31 & 38.50 \\
\hline $32 X-3$ & 299.38 & 50.0 & 42.77 & 85.53 & 1344 & 234 & 117 & 1356 & 1.65 & 0.30 & 38.67 \\
\hline $32 X-4$ & 300.88 & 50.0 & 44.14 & 88.29 & 1337 & 204 & 181 & 1155 & 1.57 & 0.00 & 38.85 \\
\hline $32 X-5$ & 302.38 & 50.0 & 41.39 & 82.78 & 1305 & 157 & 157 & 1268 & 1.80 & 0.49 & 39.03 \\
\hline $32 X-6$ & 303.88 & 49.9 & 32.14 & 64.41 & 1400 & 358 & 109 & 1633 & 1.70 & -0.34 & 39.20 \\
\hline $33 X-1$ & 306.08 & 49.8 & 39.64 & 79.60 & 1766 & 214 & 126 & 1022 & 2.06 & -0.24 & 39.46 \\
\hline $33 X-2$ & 307.58 & 50.3 & 44.77 & 89.00 & 1206 & 190 & 78 & 1094 & 1.76 & -0.22 & 39.63 \\
\hline $33 X-3$ & 309.88 & 49.8 & 44.14 & 88.64 & 1223 & 193 & 79 & 1303 & 1.58 & 0.22 & 40.00 \\
\hline $33 X-4$ & 310.58 & 50.5 & 44.14 & 87.41 & 1144 & 193 & 57 & 1337 & 1.78 & 0.23 & 40.16 \\
\hline $33 X-5$ & 312.08 & 50.0 & 44.51 & 89.04 & 1011 & 90 & 146 & 1499 & 1.85 & 0.74 & 40.51 \\
\hline $34 X-1$ & 315.78 & 50.5 & 42.39 & 83.94 & 1062 & 153 & 118 & 1510 & 1.78 & 0.13 & 41.36 \\
\hline $34 X-2$ & 317.28 & 50.0 & 41.39 & 82.78 & 1123 & 205 & 157 & 1570 & 1.74 & 0.22 & 41.70 \\
\hline $34 X-3$ & 318.78 & 50.2 & 42.39 & 84.45 & 1062 & 177 & 153 & 1510 & 1.60 & 0.22 & 42.05 \\
\hline $34 X-4$ & 320.18 & 50.0 & 39.64 & 79.28 & 996 & 189 & 88 & 1690 & 1.70 & 0.71 & 42.37 \\
\hline $34 X-5$ & 321.78 & 50.0 & 45.14 & 90.29 & 676 & 122 & 76 & 1418 & 1.65 & 0.67 & 42.74 \\
\hline $34 X-6$ & 323.28 & 49.9 & 43.39 & 86.96 & 749 & 196 & 115 & 1821 & 1.72 & 0.94 & 43.08 \\
\hline $35 \mathrm{X}-1$ & 325.38 & 50.0 & 44.77 & 89.54 & 849 & 145 & 112 & $?$ & 1.60 & 0.17 & 43.57 \\
\hline $35 X-3$ & 328.38 & 50.1 & 41.02 & 81.87 & 841 & 158 & 24 & 1621 & 1.77 & 0.16 & 44.26 \\
\hline $35 X-4$ & 329.88 & 49.9 & 39.89 & 79.94 & 953 & 138 & 63 & 1579 & 1.76 & 0.33 & 44.51 \\
\hline $35 X-4$ & 329.88 & 50.6 & 42.02 & 83.04 & 904 & 178 & 24 & 1535 & 1.76 & 0.33 & 44.51 \\
\hline $35 X-4$ & 329.88 & 50.4 & 41.64 & 82.62 & 949 & 156 & 60 & 1537 & 1.76 & 0.33 & 44.51 \\
\hline $35 X-4$ & 329.88 & 49.9 & 41.02 & 82.20 & 926 & 134 & 61 & 1548 & 1.76 & 0.33 & 44.51 \\
\hline $35 X-5$ & 331.38 & 49.8 & 39.89 & 80.10 & 990 & 163 & 125 & 1529 & 1.81 & 0.25 & 44.70 \\
\hline $35 X-6$ & 332.9 & 50.0 & 41.02 & 82.03 & 792 & 134 & 122 & 1682 & 1.64 & 0.47 & 44.89 \\
\hline $36 \times-1$ & 335.08 & 50.0 & 39.64 & 79.28 & 820 & 164 & 25 & 1766 & 1.71 & 0.52 & 45.17 \\
\hline $36 \times-2$ & 336.58 & 50.2 & 41.02 & 81.71 & 926 & 183 & 122 & 1646 & 1.79 & 0.31 & 45.36 \\
\hline
\end{tabular}


Table 1 (continued).

\begin{tabular}{cccccccccccc}
\hline Core, section & $\begin{array}{c}\text { Depth } \\
(\mathrm{mbsf})\end{array}$ & $\begin{array}{c}\mathrm{Wt} \\
(\mathrm{mg})\end{array}$ & $\begin{array}{c}\mathrm{CaCO}_{3} \\
(\mathrm{mg})\end{array}$ & $\begin{array}{c}\mathrm{CaCO}_{3} \\
(\mathrm{wt} \%)\end{array}$ & $\begin{array}{c}\mathrm{Sr} \\
(\mathrm{ppm})\end{array}$ & $\begin{array}{c}\mathrm{Mn} \\
(\mathrm{ppm})\end{array}$ & $\begin{array}{c}\mathrm{Fe} \\
(\mathrm{ppm})\end{array}$ & $\begin{array}{c}\mathrm{Mg} \\
(\mathrm{ppm})\end{array}$ & ${ }^{13} \mathrm{C}$ & $\delta^{18} \mathrm{O}$ & $\begin{array}{c}\text { Age } \\
(\mathrm{Ma})\end{array}$ \\
\hline $115-709 \mathrm{C}-$ - (Cont) & & & & & & & & & & & \\
& & & & & & & & & & & \\
36X-3 & 338.08 & 49.8 & 41.02 & 82.36 & 792 & 183 & 61 & 1646 & 1.71 & 0.25 & 45.55 \\
37X-1 & 344.88 & 49.9 & 37.52 & 75.18 & 920 & 227 & 93 & 1546 & 1.58 & 0.04 & 46.41 \\
37X-2 & 346.38 & 50.3 & 38.89 & 77.32 & 836 & 219 & 64 & 1736 & 1.54 & 0.02 & 46.60 \\
37X-3 & 347.88 & 50.8 & 34.39 & 67.69 & 874 & 218 & 29 & 1634 & 1.59 & 0.18 & 46.79 \\
37X-4 & 349.38 & 50.0 & 33.64 & 67.28 & 1026 & 163 & 74 & 1680 & 1.66 & 0.34 & 46.98 \\
37X-5 & 350.88 & 50.9 & 40.64 & 79.85 & 886 & 160 & 62 & 1513 & 1.55 & 0.33 & 47.18 \\
37X-6 & 352.38 & 51.0 & 42.39 & 83.12 & 637 & 177 & 83 & 1757 & 1.48 & 0.60 & 47.37 \\
\hline
\end{tabular}

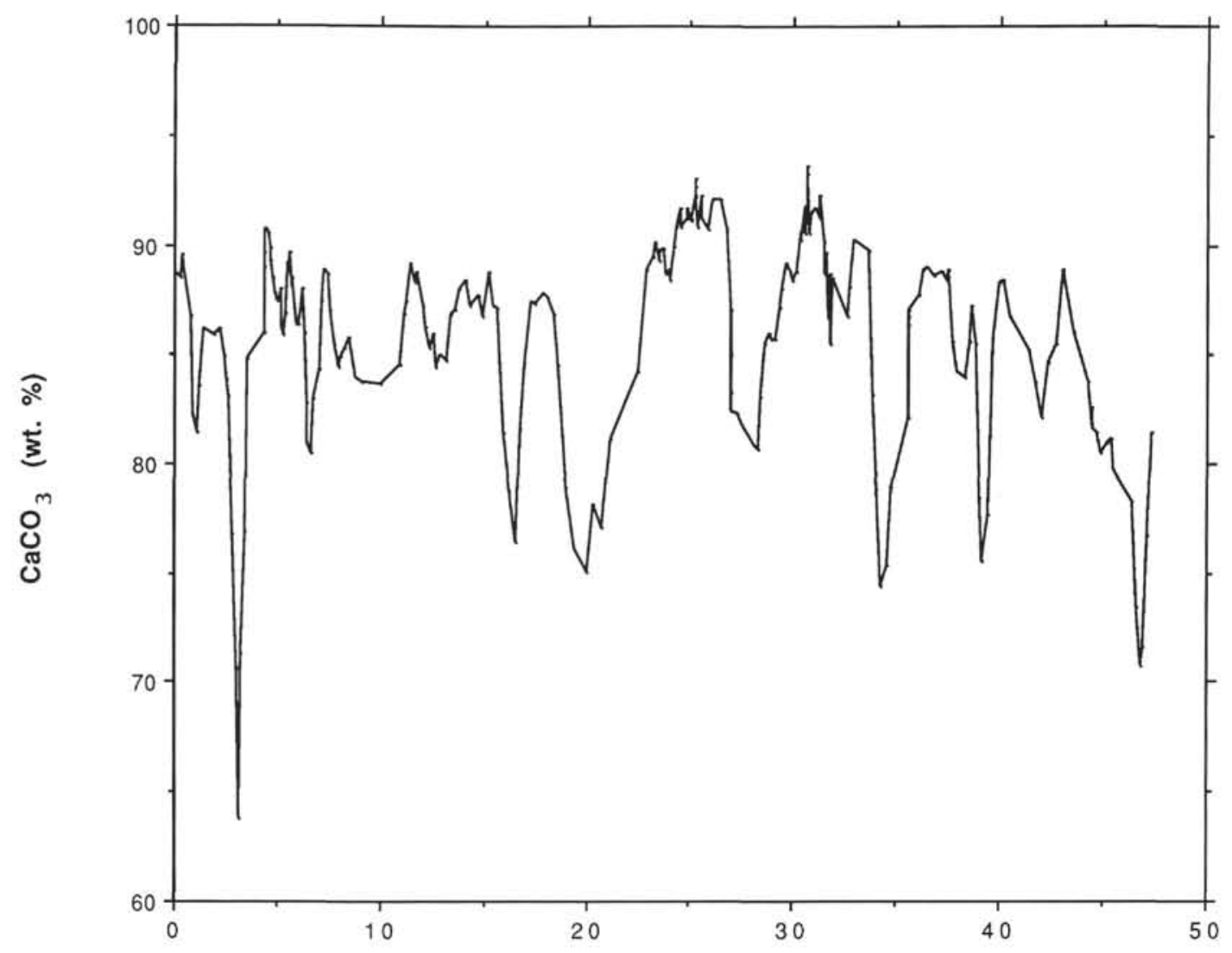

Age (Ma)

Figure 1. Three-point moving average of $\mathrm{CaCO}_{3}(\mathrm{wt} \%)$ in the fine fraction of sediments vs. age.

that we can rule out noncarbonate sources of strontium during leaching because of our sample cleaning procedures.

Changes in the $\mathrm{Sr} / \mathrm{Ca}$ ratio of carbonate sediments can be ascribed either to changes in the distribution coefficient of strontium in calcite $\left(D_{c}\right)$, thought to be primarily caused by temperature changes, or to the $\mathrm{Sr} / \mathrm{Ca}$ ratio in seawater, $(\mathrm{Sr} / \mathrm{Ca})_{s w}$ :

$$
(\mathrm{Sr} / \mathrm{Ca})=D_{c}(\mathrm{Sr} / \mathrm{Ca})_{s w}
$$

Experimental work has shown that strontium uptake during nonbiogenic calcite growth is not strongly temperature dependent (Katz et al., 1972; Baker et al., 1982); however, there is conflicting evidence on the importance of temperature dependence on the strontium content of foraminiferal calcite. Delaney et al. (1985) found little correlation between seawater temperature and strontium concentrations in either core-top planktonic foraminifers or in laboratory-cultured foraminifers. On the other hand, Cronblad and Malmgren (1981) observed large and consistent glacial/interglacial variations of strontium and magnesium in foraminifers from Pleistocene sediments near the Polar Front in the southern Indian Ocean. The positive correlation they observed between warm-water climatic indexes and strontium concentrations in planktonic foraminifers strongly suggests a temperature control on the strontium.

Graham et al. (1982) suggested that strontium concentration variations preserved in ancient sediments may reflect original variations in the $\mathrm{Sr} / \mathrm{Ca}$ ratio in ancient seawater. On a much larger time scale, Renard (1986) attempted to correlate strontium and magnesium concentration changes in bulk pelagic carbonate sediments from many different localities and ascribed these changes to $\mathrm{Sr} / \mathrm{Ca}$ and $\mathrm{Mg} / \mathrm{Ca}$ secular variation in seawater. Recently, Boyle and Keigwin (1982) and Lea and Boyle 


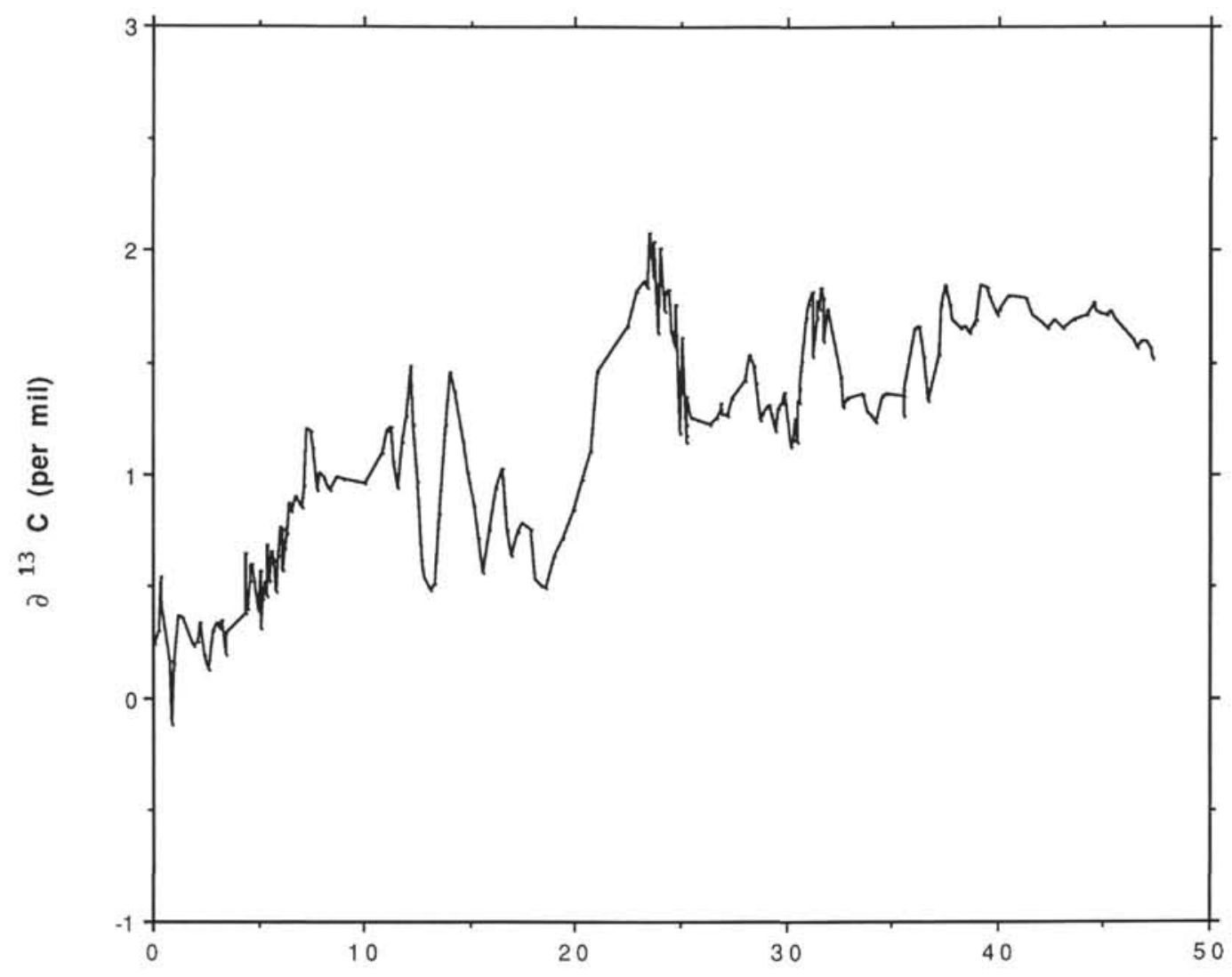

Age (Ma)

Figure 2. Three-point moving average of the carbon isotopic ratios in the carbonate fine fraction vs. age.

(1989) have convincingly shown that variations in seawater barium and cadmium concentrations are recorded by benthic foraminifers. Although neither strontium nor magnesium show very significant nonconservative behavior in modern-day seawater, past variations in their concentrations, relative to calcium concentrations, can be expected to be recorded by carbonate-producing organisms.

Graham et al. (1982) proposed two major mechanisms for changing oceanic $\mathrm{Sr} / \mathrm{Ca}$ ratios: (1) variable hydrothermal input of calcium to the oceans at the ridge crests and (2) variable rates of strontium removal resulting from changes in the proportion of high-strontium aragonite vs. low-strontium calcite sedimentation. The ratio of aragonite to calcite should increase during high sea-level stands when more shallow carbonate banks are flooded. Graham et al. (1982) concluded that both processes played important roles in determining Cenozoic $\mathrm{Sr} / \mathrm{Ca}$ ratios. Delaney and Boyle (1986) concluded that for the past 40 m.y. both $\mathrm{Li} / \mathrm{Ca}$ and $\mathrm{Sr} / \mathrm{Ca}$ ratios in foraminifers changed very little and that lack of variability in the $\mathrm{Li} / \mathrm{Ca}$ ratio implied that there has been no significant variability in oceanic hydrothermal fluxes. They also concluded that over the past $100 \mathrm{~m}$.y. there has been less than a factor of 2 decrease in hydrothermal chemical fluxes. On the other hand, Renard (1986) has reported numerous larger variations of $\mathrm{Sr} / \mathrm{Ca}$ and $\mathrm{Mg} / \mathrm{Ca}$ ratios in bulk pelagic carbonate samples and once again attributed these variations to both hydrothermal and sea-level variations.

Increased hydrothermal activity should bring about an increase in oceanic-dissolved strontium but a larger increase in oceanic-dissolved calcium; hence, the $\mathrm{Sr} / \mathrm{Ca}$ ratio of seawater should decrease (Palmer and Edmond, 1989). Likewise, increased hydrothermal activity is thought to cause global sea-level rise, in which case more aragonite sedimentation would be expected on shallow shelves and bank tops and the pelagic $\mathrm{Sr} / \mathrm{Ca}$ of seawater should decrease.

Strontium concentration is a useful indicator of carbonate diagenesis because foraminifers and, especially, nannofossils precipitate calcite that is enriched in strontium relative to equilibrium calcite. During burial diagenesis, some of the biogenic calcite is dissolved and reprecipitated. In the process, strontium is released into the pore fluids (Baker et al., 1982) and diagenesis results in a lower concentration of strontium in older carbonate sediments.

The potential importance of diagenesis in determining the observed strontium concentrations (Fig. 3) can be evaluated with the pore-water strontium analyses for Site 709 reported by Swart and Burns (in this volume). From their data, we can estimate a present-day, near-surface, dissolved strontium gradient of about $4.2 \times 10^{11} \mathrm{~mol} / \mathrm{cm}^{4}$. Using Fick's Law and a diffusion coefficient in the sediments of about $3 \times 10^{-6} \mathrm{~cm}^{2} / \mathrm{s}$, we can calculate a diffusive flux of strontium out of sedimentary pore waters into the overlying seawater of $4.0 \times 10^{-3} \mathrm{~mol} / \mathrm{cm}^{2} / \mathrm{m} . \mathrm{y}$. Compare this to the rate of accumulation of strontium in the sediments (at $75 \%$ porosity, $90 \% \mathrm{CaCO}_{3}, 2100 \mathrm{ppm} \mathrm{Sr}, 10 \mathrm{~m} / \mathrm{m}$.y. sedimentation rate): $1.94 \times 10^{-2} \mathrm{~mol} / \mathrm{cm}^{2} / \mathrm{m}$.y. (note that this is $\mathrm{cm}^{2}$ of pore water). Thus, about $20 \%$ of all the strontium that accumulates on the seafloor diffuses back into overlying seawater (and, at the very least, $20 \%$ of the carbonate has been recrystallized). However, as shown previously (Baker, 1985; Rich- 


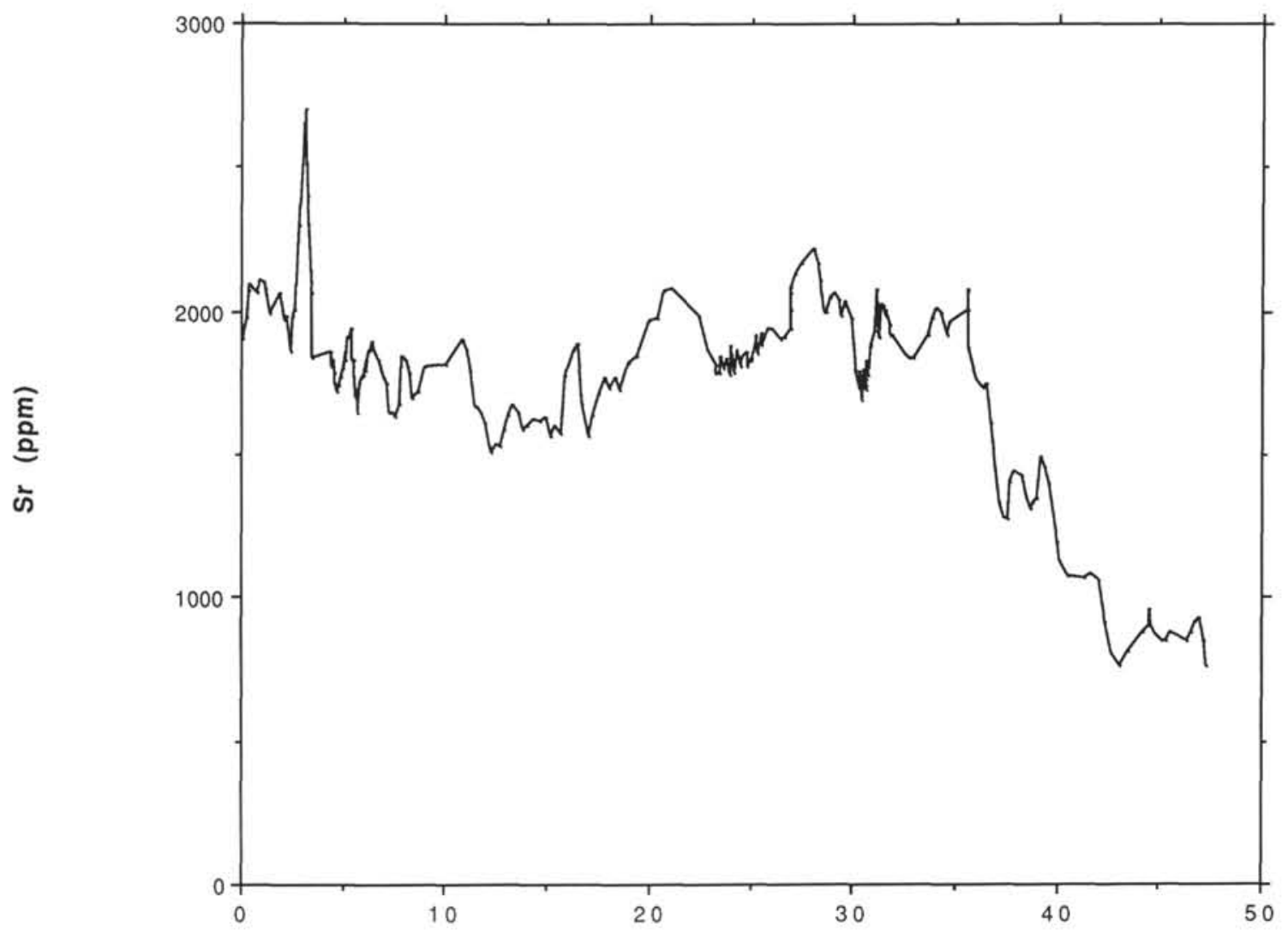

Age (Ma)

Figure 3. Three-point moving average of the strontium concentrations in the carbonate portion of the fine fraction vs. age.

ter and DePaolo, 1988), this number is probably closer to $50 \%$ recrystallized within about $10 \mathrm{~m} . \mathrm{y}$. of deposition. Almost all of this strontium is lost from the upper $100 \mathrm{~m}$ of sediment, since below this depth there is no observed pore-water strontium concentration gradient (Swart and Burns, this volume). If we assume that the present-day average strontium concentration in the carbonate fine fraction of $2100 \mathrm{ppm}$ was typical of sediments for the last several million years, then these calculations give us the result that after about 7-m.y. (the age at $100 \mathrm{~m}$ below seafloor [mbsf]) of diagenesis there should be a decrease to about $1680 \mathrm{ppm}$. In fact, this is approximately the magnitude of concentration change observed in sediments from $7 \mathrm{Ma}$ to the present.

The factor that seems most likely to change the dissolved strontium gradient out of the sediments and the resulting diagenetic strontium loss of ancient sediments is bottom-water (hence, pore-water) temperature. Temperature can affect the strontium distribution coefficient in calcite and the diffusion coefficient of strontium in sediments, but the most thermally activated process is the rate of calcite recrystallization. For example, assuming that a $10^{\circ} \mathrm{C}$ rise in bottom-water temperature can produce a doubling of the rate of calcite dissolution and reprecipitation, then we could expect roughly a doubling of the rate of strontium release to the pore waters and a doubling of the diffusive flux of strontium to overlying seawater. The net result should be lower strontium concentrations in the sediments during periods of higher bottom-water temperature.

There is a feedback mechanism to the above-mentioned process. At the present time, calcite produced during diagenesis be- low 100 mbsf should have about $1160 \mathrm{ppm}$ strontium (given the average $\mathrm{Sr} / \mathrm{Ca}$ ratio of pore waters observed below $100 \mathrm{mbsf}$ and a distribution coefficient of strontium in calcite of 0.04 ; Baker, 1985). Clearly, except in the Eocene, diagenetic calcite probably constitutes only about $50 \%$ of the total fine fraction (assuming for the moment an original strontium content of $2000 \mathrm{ppm}$ ). If rates of recrystallization are sufficiently accelerated, the $\mathrm{Sr} / \mathrm{Ca}$ ratio of pore waters would be elevated, the diagenetic calcite would have a higher strontium content, and there would be a decreased loss of strontium from the sediments.

In summary, diagenesis has been demonstrably important in influencing the strontium content of the carbonate sediments at Site 709 , but original variations of the strontium concentrations of the carbonate, caused by paleotemperature changes or changes in seawater $\mathrm{Sr} / \mathrm{Ca}$ ratios, could persist. In the simple case where $50 \%$ of the biogenic calcite has been replaced by diagenetic calcite of a fixed composition, the original concentration variation would be halved by diagenesis. Accordingly, to crudely deconvolve the strontium curve shown in Figure 3, we should do the following:

1. Fix the modern-day ratio at the observed value.

2. Back to $7 \mathrm{Ma}$, adjust the observed values for linearly increasing amounts of diagenetic calcite to $50 \%$ calcite of 1160 ppm strontium by $7 \mathrm{Ma}$.

3. Before $7 \mathrm{Ma}$, any carbonate with greater than $1580 \mathrm{ppm}$ originally had $>2000 \mathrm{ppm}$ and any carbonate with $<1580 \mathrm{ppm}$ originally had $<2000 \mathrm{ppm}$. If we assume a $50 \%$ addition of diagenetic carbonate of $1160 \mathrm{ppm}$, then the following simple al- 


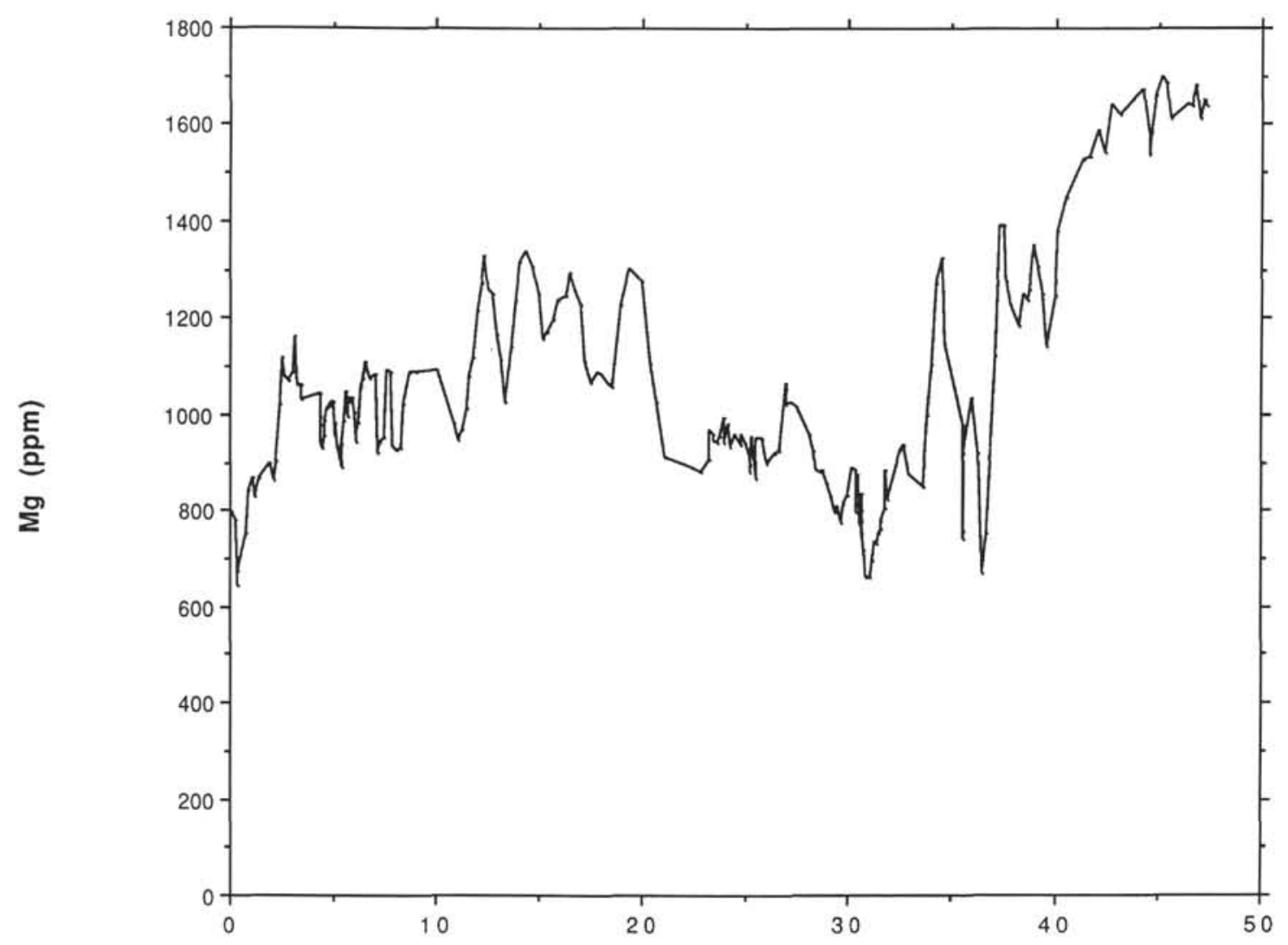

Age (Ma)

Figure 4. Three-point moving average of the magnesium concentrations in the carbonate portion of the fine fraction vs. age.

gorithm can be used to calculate the original strontium content of carbonate $\left(\mathrm{Sr}_{i}\right)$ from the present-day observed value $(\mathrm{Sr})$ :

$$
2 \times \mathrm{Sr}-1160=\mathrm{Sr}_{i} \text { (in ppm) }
$$

4. Note that we have not accounted for any variation of rates of diagenetic strontium loss and we have assumed steadystate conditions in the pore waters throughout.

The deconvolved strontium concentration curve (Fig. 8) is quite similar to the results published by Graham et al. (1982) for $\mathrm{Sr} / \mathrm{Ca}$ ratios of planktonic foraminifers. The most striking feature of both studies, also noted by Renard (1986) and apparent in Delaney and Boyle (1988), is the late Eocene strontium increase. This feature coincides with the late Eocene initiation of the rise in the ${ }^{87} \mathrm{Sr} /{ }^{86} \mathrm{Sr}$ ratio in seawater (Elderfield, 1986). It also coincides with the decrease in seafloor spreading rates (Pitman, 1978) and the lowering of sea level from middle Eocene highs (Haq et al., 1987).

Elsewhere, strontium concentrations display less dramatic variations, but there seems to be some correlation between high sea level stands and low strontium concentrations, and vice versa. For example, some of the lowest sea levels of the Neogene were thought to have occurred during the middle and late Miocene centered at about $10 \mathrm{Ma}$. A strontium peak also occurs at this time. Low sea levels in the late Oligocene coincide with high strontium concentrations. Low sea levels in the late Pliocene and Pleistocene coincide with high strontium concentrations.
This implies that sea level is important, but it does not distinguish between the hydrothermal and shallow-water aragonitic causes.

What fraction of the strontium concentration change can reasonably be explained by variations in the aragonite/calcite ratio? This question was originally addressed by Graham et al. (1982) and it is their model, with a minor adaptation, that we apply below to look again at this problem.

The oceanic mass balance for strontium can be written as:

$$
d \mathrm{Sr} / d t=F_{D}+F_{R}-F_{S},
$$

where $F$ is the flux of strontium to the ocean and subscripts $D$, $R$, and $S$ represent the diagenetic, riverine, and carbonate sedimentation fluxes of strontium, respectively. The value, $F_{D}$, was discussed by Elderfield and Gieskes (1982). As noted by Graham et al. (1982), $F_{S}$ is given by:

$$
F_{S}=f_{A} F_{A}+\left(1-f_{A}\right) F_{C},
$$

where $F_{A}$ and $F_{C}$ are the sedimentary fluxes of aragonite and calcite, respectively, and $f_{A}$ is the fraction of aragonite.

Furthermore,

$$
F_{S}=f_{A} D_{A}(\mathrm{Sr} / \mathrm{Ca})_{s w} J+\left(1-f_{A}\right) D_{C}(\mathrm{Sr} / \mathrm{Ca})_{s w} J,
$$

where $J$ is the burial flux of carbonate sediments. We have assumed values of $D_{A}=1.00$ and $D_{C}=0.23$ from present-day 


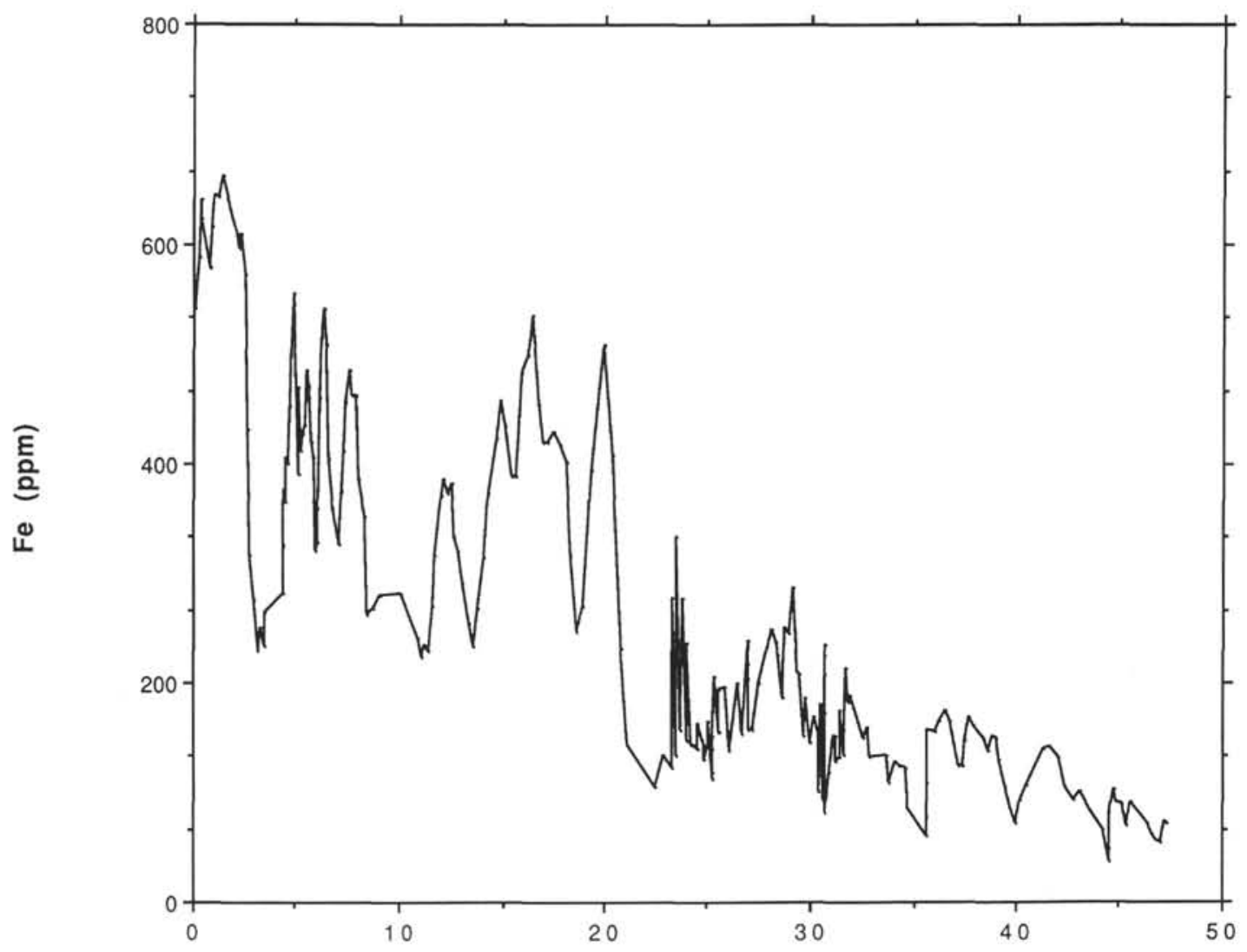

\section{Age (Ma)}

Figure 5. Three-point moving average of the iron concentrations in the carbonate portion of the fine fraction vs. age.

values of the $\mathrm{Sr} / \mathrm{Ca}$ ratio of seawater, aragonitic benthic green algae and corals, and bulk pelagic carbonate sediments. Here we take

$$
F_{D}=(0.2) D_{C}(\mathrm{Sr} / \mathrm{Ca})_{\mathrm{SW}} J
$$

because, as discussed above, about $20 \%$ of the strontium in modern carbonate sediments is returned to overlying seawater (and possibly higher in aragonitic sediments). Then, at steady state

$$
F_{R} / J(\mathrm{Sr} / \mathrm{Ca})_{s w}=0.77 f_{A}+0.184
$$

At the present time,

$$
\left(F_{D}+F_{R}\right) / J=0.0025,
$$

which can be adjusted to make the present-day $f_{A}$ come out at the most appropriate value. Substituting for $F_{D}$, we find that

$$
F_{R} / J=0.0021
$$

which is very close to the estimate of Delaney and Boyle (1988),

We can assume, for the sake of discussion, that this ratio $\left(F_{R} / J\right)$ has been constant throughout time. This allows us to evaluate independently the importance of changes in $f_{A}$ :

$$
f_{A}=0.00273 /(\mathrm{Sr} / \mathrm{Ca})_{s w}-0.239 \text {. }
$$

The results of this calculation are given in Table 2. One can see that late Eocene values of $1000 \mathrm{ppm}$ strontium $(\mathrm{Sr} / \mathrm{Ca}=$ .00114 ) imply that about $30 \%$ of the total $\mathrm{CaCO}_{3}$ was deposited as high-strontium aragonite. During the late Oligocene sea-level low stand (around $28 \mathrm{Ma}$ ), the high values of $\mathrm{Sr} / \mathrm{Ca}$ imply a value of $f_{A}=0$ (i.e., there was no high-strontium aragonite; most shallow carbonate-producing banks must have been subaerially exposed). The model is adjusted to make modern values of high-strontium aragonite equal a reasonable value of total $\mathrm{CaCO}_{3}$ sedimentation.

With regard to strontium concentration variations, we conclude the following. First, diagenesis is clearly of importance in decreasing the strontium concentrations of carbonate sediments. Nevertheless, real paleoceanographically controlled variations are preserved to some degree. The reasonable correlation between high sea-level stands and low strontium values suggests some kind of sea-level control, most likely through a shelf/basin fractionation mechanism. High sea-level stands resulted in larger fractions of aragonitic sediments and less strontium in seawater. As pointed out by Delaney and Boyle (1986), a similar shelf/basin fractionation mechanism can also account for the secular changes observed in the carbonate compensation depth (CCD). Variations in hydrothermal fluxes may also produce changes in the oceanic Sr/Ca ratio, but Delaney and Boyle (1986) concluded that these changes were insufficient to account for more than a fraction of the observed strontium variations. $\mathrm{Pa}$ leotemperature changes deduced from the oxygen isotopic record are also probably insufficient to account for the observed variations of strontium. 


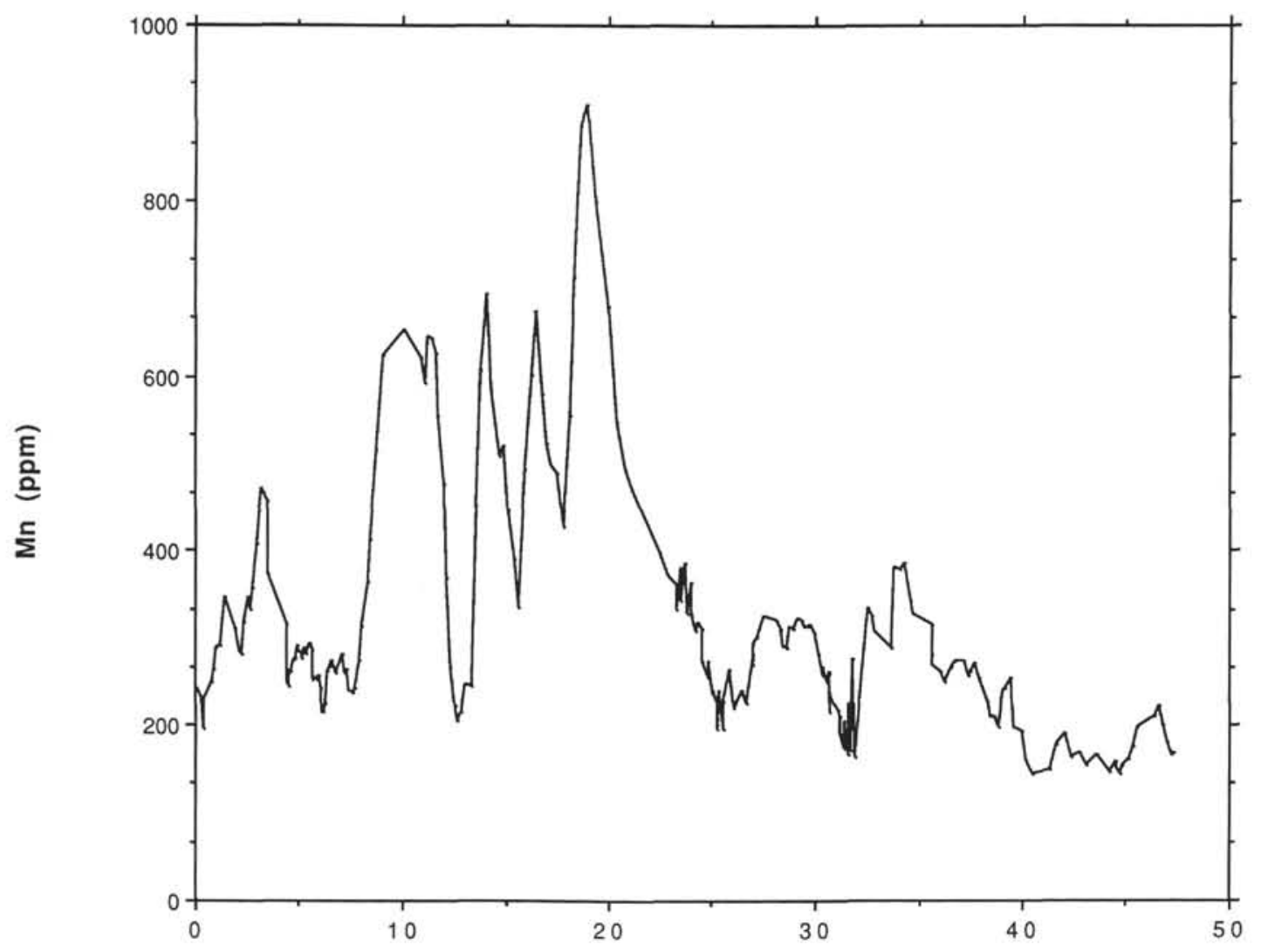

Age (Ma)

Figure 6. Three-point moving average of the manganese concentrations in the carbonate portion of the fine fraction vs. age.

\section{Oxygen Isotopes}

If it is correct, as deduced above, that diagenetic calcite constitutes about $50 \%$ of the carbonate sediments, then this should be reflected in the oxygen isotopic composition of the fine fraction. In Figure 9, the approach of the carbonate sediments to apparent oxygen isotopic equilibration with pore waters is illustrated. For the sake of these calculations it was assumed that (1) the $\delta^{18} \mathrm{O}$ of pore waters was constant at $0.0 \%$ (SMOW) (near the present-day values measured by P. K. Swart, pers. comm., 1989), (2) the bottom-water temperature was $2^{\circ} \mathrm{C}$, and (3) the average geothermal gradient at this site was about $35^{\circ} \mathrm{C} / \mathrm{km}$. The oxygen isotopic fractionation factor was calculated from the equation of O'Neil et al. (1969). From these calculations, we can safely conclude that the course of burial diagenesis throughout the history of Site 709 would have involved an increase in $\delta^{18} \mathrm{O}$ of the carbonate sediments. This is particularly evident if we assume, following Savin (1977), that before $15 \mathrm{Ma}$ the oceans were about $1 \% 0$ lower in $\delta^{18} \mathrm{O}$ than at present (of course, the pore waters before $15 \mathrm{Ma}$ also would have been isotopically lighter).

A comparison of the oxygen isotopic data (Fig. 7) with the strontium concentration data (Fig. 3) reveals an inverse correlation: almost invariably, lower strontium values are associated with higher oxygen isotopic compositions. This relationship is most clearly revealed in the Eocene chalks. Because similar coincident variations would be expected to result from temperature changes (colder temperatures should result in heavier oxygen isotopic ratios and lower strontium concentrations) and diagenetic changes, it is not straightforward to differentiate between diagenetic and paleoclimatic causes for the observed relationship.

\section{Magnesium}

$\mathrm{The} \mathrm{Mg} / \mathrm{Ca}$ ratios in carbonate sediments respond to the same factors that influence $\mathrm{Sr} / \mathrm{Ca}$ ratios, namely, the temperature of precipitation, the $\mathrm{Mg} / \mathrm{Ca}$ ratio of ancient seawater, and diagenesis. Cronblad and Malmgren (1981) showed an apparent positive correlation between near-surface, ocean-water temperature and magnesium concentrations in planktonic foraminifers. The range of observed concentrations was from about 400 to $800 \mathrm{ppm}$. Temperature controls, therefore, should bring about a correlation between strontium and magnesium concentrations (which we did not observe). If sea-level changes can cause a secular variation of aragonite/calcite ratios, they should also bring about a similar variation of the ratio high-magnesium calcite/ calcite. Because high-magnesium calcite is usually much less abundant in shallow-water carbonate sediments than aragonite, however, these variations would not be very effective in altering seawater $\mathrm{Mg} / \mathrm{Ca}$ values. Hydrothermal input should have a strong influence on the seawater $\mathrm{Mg} / \mathrm{Ca}$ ratio and in the same direction as the seawater $\mathrm{Sr} / \mathrm{Ca}$ ratio. Because of the longer residence time of magnesium than strontium, however, maxima (or minima) in $\mathrm{Mg} / \mathrm{Ca}$ ratios should follow the maxima (or minima) of Sr/Ca ratios by a few million years (e.g., Renard, 1986), 


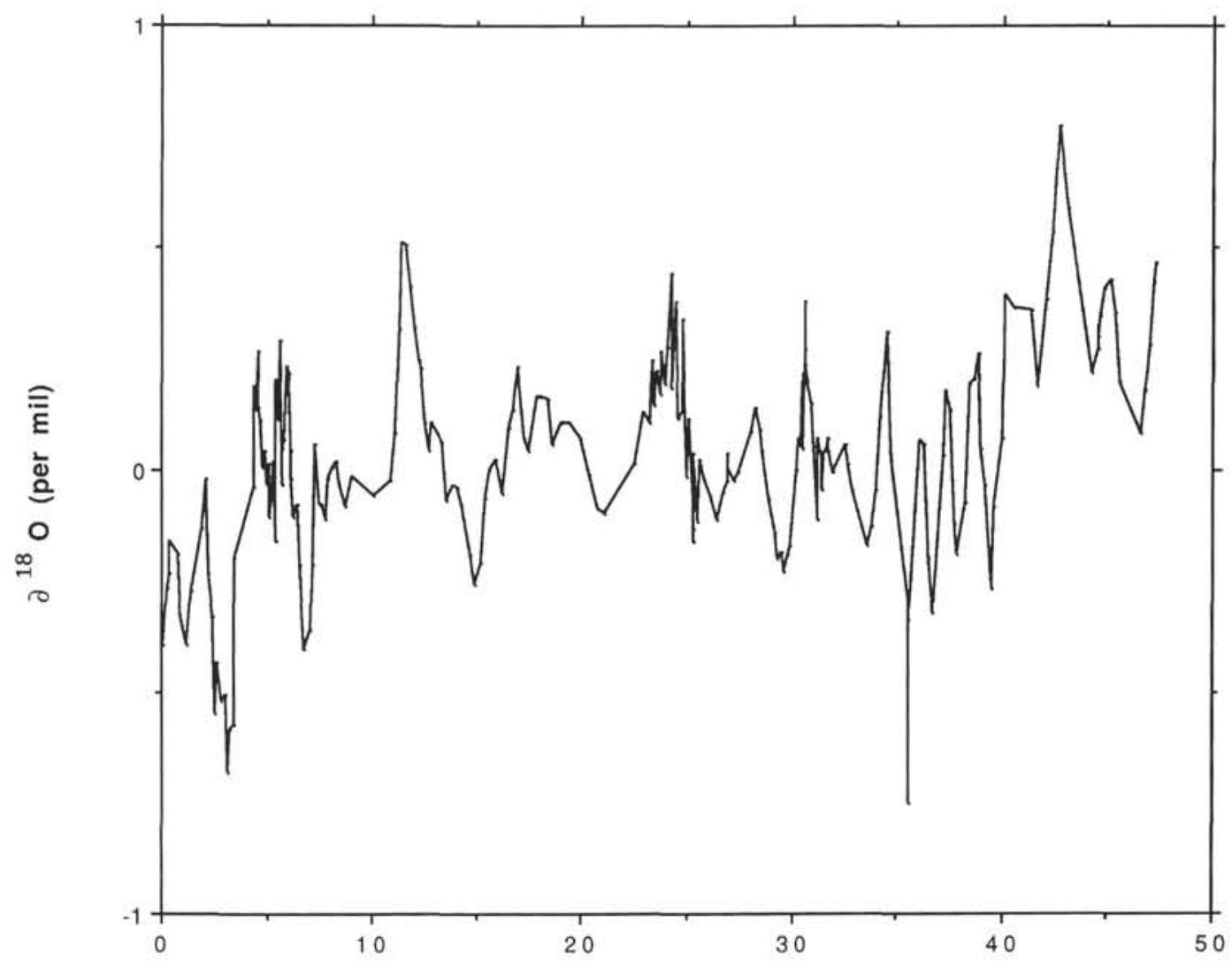

Age (Ma)

Figure 7. Three-point moving average of the oxygen isotopic ratios in the carbonate fine fraction vs. age.

and variations should generally be positively correlated. Finally, diagenesis should bring about an increase in the $\mathrm{Mg} / \mathrm{Ca}$ and a decrease in the $\mathrm{Sr} / \mathrm{Ca}$ ratio of pelagic carbonate sediments (e.g., Baker et al., 1982), thus producing an inverse correlation between strontium and magnesium concentrations.

In comparing Figures 3 and 4, we observe a tendency for strontium and magnesium to be inversely correlated. This is best observed at the top of the core (in the last 3 m.y.) where diagenetic recrystallization of calcite occurs at a maximum rate and near the bottom of the core (from about 35 to $47 \mathrm{Ma}$ ). We therefore think that a major portion of the changes observed in the $\mathrm{Mg} / \mathrm{Ca}$ and $\mathrm{Sr} / \mathrm{Ca}$ ratios of the carbonate fine fraction are of diagenetic origin. Nevertheless, as mentioned previously, diagenesis should not eliminate original variations; rather, it should only dampen their observed amplitudes.

\section{Iron and Manganese}

Iron (Fig. 5) and manganese (Fig. 6) are both transition metals with similar ionic radii and charges; thus, both would be expected to have similar distribution coefficients in calcite. Both elements display variable redox states in seawater and shallow pore waters. Manganese (II) is thought to be the dominant redox state of manganese in seawater (even though it is thermodynamically unstable), whereas iron (III) is thought to be dominant for iron (Bruland, 1983). Neither element is well mixed in seawater. Generally, manganese is more abundant than iron, particularly in surface waters (Bruland, 1983). Both iron and manganese are greatly enriched in pore waters as a result of microbial oxidation of organic carbon. Although low levels of manganese and iron may be incorporated into biogenic calcite, it seems much more likely that diagenesis will play the greatest role in their incorporation into carbonate sediments. Because manganese is reduced more easily and rapidly than oxidized iron, pore-water maxima of these elements are attained at different sub-bottom depths (e.g., Froehlich et al., 1979). Manganese is also generally more abundant than iron in pore waters (Froehlich et al., 1979; Sawlan and Murray, 1983). At Site 709 there is good evidence for sulfate reduction in the pore waters (Swart and Burns, this volume) and pyrite precipitation in the sediments; hence, dissolved iron concentrations in the pore waters should only be high in the upper few meters of the sediment column.

From the above discussion, we conclude that it is most likely that iron and manganese concentrations in pelagic carbonate sediments are controlled by diagenetic processes. Their microbial reduction allows them to be solubilized and made available for incorporation into reprecipitated diagenetic calcite or perhaps into trace amounts of a mixed iron, manganese, magnesium, and calcium carbonate phase. Because of their slightly different redox chemistries, peak concentrations of these elements in the carbonate phase may be offset in terms of sub-bottom depth, and concentration peaks for both elements may not exactly coincide.

The manganese data (Fig. 6) may be the best means for assessing the nature of the slumping process described by D. Rio and H. Okada (Shipboard Scientific Party, 1988b, p. 468). They determined that the interval between Sections 115-709C-15H-4 (136 mbsf) and 115-709C-18H-3 (164 mbsf) was disturbed. Al- 


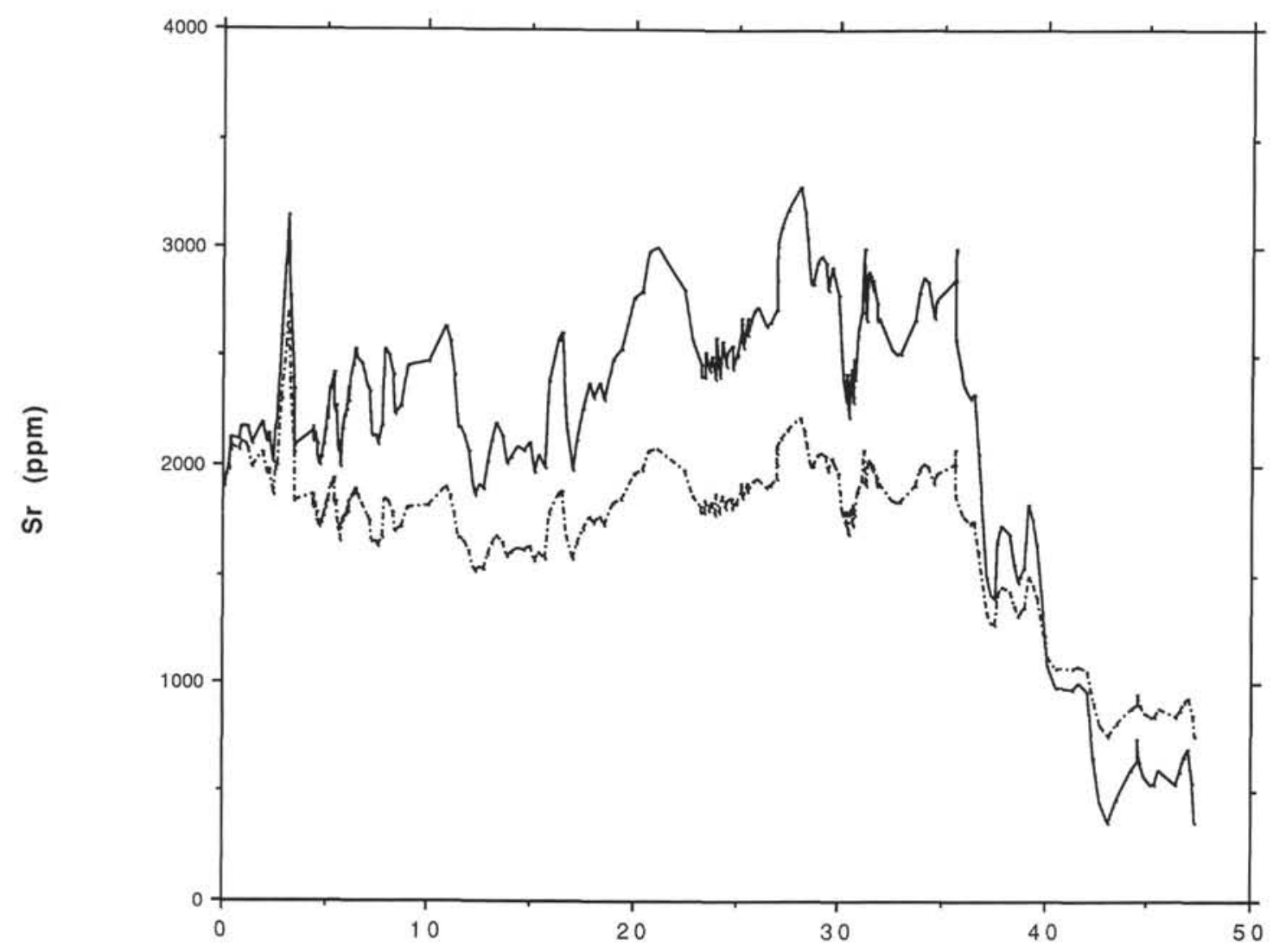

Figure 8. Three-point moving average of the deconvoluted strontium values (line) and strontium three-point moving average (dashed line) vs. age.

Table 2. The aragonitic fraction of the total carbonate sedimentation calculated to be necessary to explain the observed $\mathrm{Sr}$ / Ca ratio of pelagic carbonate sediments.

\begin{tabular}{ccc}
\hline$(\mathrm{Sr} / \mathrm{Ca})_{s w}$ & $(\mathrm{Sr} / \mathrm{Ca})$ & $f_{A}$ \\
\hline 0.0030 & 0.00069 & 0.67 \\
0.0050 & 0.00115 & 0.31 \\
0.0080 & 0.00184 & 0.10 \\
0.0087 & 0.00200 & 0.07 \\
0.0100 & 0.00230 & 0.03 \\
0.0110 & 0.00250 & 0.01
\end{tabular}

Note: $(\mathrm{Sr} / \mathrm{Ca})_{s w}=$ the ratio in seawater $(0.0087$ is the modern-day value); $\mathrm{Sr}$ / $\mathrm{Ca}=$ the ratio observed in pelagic carbonate sediments; and $f_{A}=$ the fraction of aragonite of the carbonate sediments.

though the whole interval was stratigraphically disordered, they concluded that individual nannofossil assemblages were coherent. Thus, reworking must have resulted from discrete slump blocks. S. Robinson (pers. comm., 1989; Shipboard Scientific Party, 1988b) used magnetic susceptibility to correlate between
Holes 709A and 709C. From his work, we can place the top of the slumped interval in Hole 709A at about 135 mbsf. Presumably, the bottom of this interval in 709A is at about 163 mbsf. This depth interval corresponds to a time interval from about 13.0 to $17.5 \mathrm{Ma}$.

We interpret the large increase in manganese concentration (Fig. 6) at 19.0 Ma and subsequent decrease at 18.1 Ma as the top of the intact sedimentary record. This corresponds closely, but not perfectly, with a large iron concentration peak and, importantly, with a sharp decrease in the ${ }^{13} \mathrm{C} /{ }^{12} \mathrm{C}$ ratio. The rapid burial of this sediment by several meters of slumped sediment created an effective barrier to the diffusion of dissolved oxygen into the pore waters and rapidly accelerated the rates of manganese and iron reduction and, possibly, of sulfate reduction. The two following manganese cycles (with peaks at 13.8 and 16.3 $\mathrm{Ma}$ in Fig. 6) are interpreted as repeated sequences with similar diagenetic histories. If the enhanced concentrations of manganese, iron, and magnesium actually indicate the precipitation of one or two separate mixed carbonate mineral phases during early diagenesis, these phases would be expected to have very low carbon isotopic compositions. The presence of small amounts of such minerals could explain the associated decreases of $\delta^{13} \mathrm{C}$ over the same depth interval. Two phases are proposed because iron and manganese correlate very closely over the slumped interval, but they do not correlate closely with magnesium and strontium. Small amounts of dolomite were identified in smear slides of sediments from the slumped interval and near the base of the hole from $44.5 \mathrm{Ma}$ to the core bottom. 


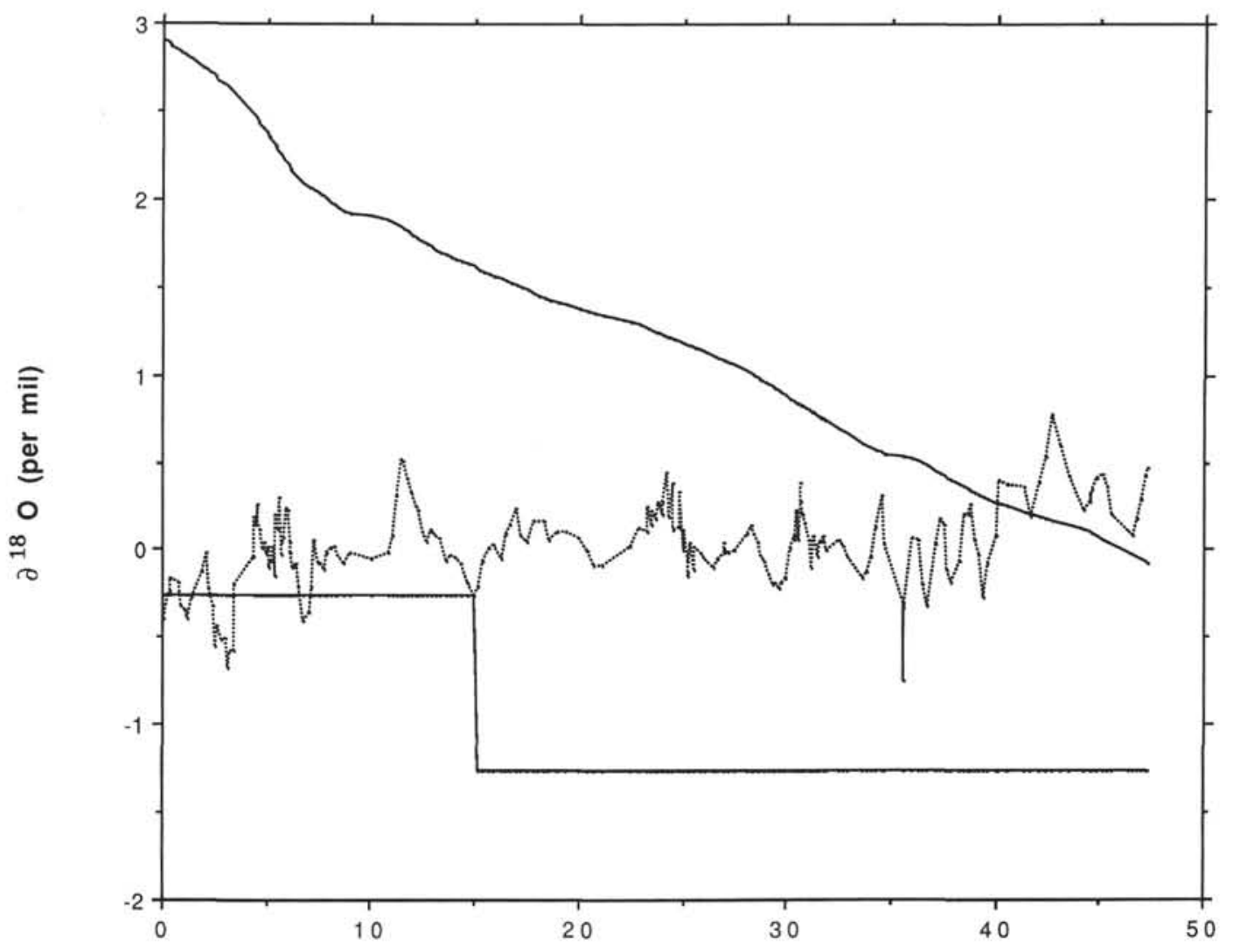

Age (Ma)

Figure 9. Oxygen isotopic composition of calcite in equilibrium with present-day pore waters, three-point moving average of the oxygen isotopic composition of the carbonate fine fraction (dotted line), and presumed initial isotopic composition of the fine fraction vs. age. See text for further details.

\section{CONCLUSIONS}

A quantitative evaluation of the mechanisms involved in producing minor element concentration variations in deep-sea carbonate sediments is not yet possible. Such determinations will require a careful comparison of several sets of chemical data measured on deep-sea carbonate sediments from different oceans and in different environmental settings. Nevertheless, we can draw some useful conclusions from the present study.

A correlation is observed between high sea-level stands and high values of $\delta^{13} \mathrm{C}$. It is suggested that this supports the hypothesis proposed by Shackleton (1987) that $\delta^{13} \mathrm{C}$ is controlled by the fraction of organic carbon that is buried: more organic carbon is buried during high stands and oceanic-dissolved inorganic carbon becomes isotopically heavier. Carbon isotopic changes mirror the long-term (Eocene to Present) decrease in sea level resulting from slower rates of seafloor spreading. Superimposed on this long-term change are several shorter term variations.

There appears to be some correlation between high sea-level stands and high $\mathrm{CaCO}_{3}$ contents in the sediment (this relationship is discussed more thoroughly elsewhere in this volume). This relationship implies a shelf-basin fractionation mechanism for carbonate accumulation: more carbonate is deposited on the shelves during high stands and the CCD rises.

Because much of the shelf carbonate is aragonite, more strontium is buried during sea-level highstands and oceanic $\mathrm{Sr} / \mathrm{Ca}$ ra- tios decrease. As a result, we observed an inverse relationship between sea level and strontium concentration.

Diagenesis plays an important role in many of the observed parameters. Burial diagenesis clearly affects strontium and magnesium concentrations and undoubtedly oxygen isotopic ratios as well. Microbially mediated oxidation of organic carbon in the slumped sediments causes early diagenetic reactions such as reduction of iron and manganese oxides, sulfate reduction, and probably precipitation of mixed carbonate minerals. This causes large variations of iron, manganese, magnesium, and carbon isotopic ratios within the slumped interval.

\section{ACKNOWLEDGMENTS}

We thank the crew and the rest of the shipboard technical and scientific parties, especially the co-chief scientists, J. Backman and R. A. Duncan. Obviously, the help of all of these people was essential for the present study. We acknowledge the support by USSAC and NSF-OCE-87-18382 (to P. A. Baker).

\section{REFERENCES}

Baker, P. A., 1985. Pore-water chemistry of carbonate-rich sediments, Lord Howe Rise, southwest Pacific Ocean. In Kennett, J. P., von der Borch, C. C., et al., Init. Repts. DSDP, 90: Washington (U.S. Govt. Printing Office), 1249-1256.

Baker, P. A., Gieskes, J. M., and Elderfield, H., 1982. Diagenesis of carbonates in deep-sea sediments-evidence from $\mathrm{Sr} / \mathrm{Ca}$ ratios and interstitial dissolved $\mathrm{Sr}^{2+}$ data. J. Sediment. Petrol., 52:71-82. 
Boyle, E. A., and Keigwin, L. D., 1982. Deep circulation of the North Atlantic over the last 200,000 years: geochemical evidence. Science, 218:784-787.

Bruland, K. W., 1983. Trace elements in sea-water. In Riley, J. P., and Chester, R. (Eds.), Chemical Oceanography (Vol. 8): London (Academic Press), 157-220.

Cronblad, H. G., and Malmgren, B. A., 1981. Climatically controlled variation of $\mathrm{Sr}$ and $\mathrm{Mg}$ in Quaternary planktonic foraminifera. $\mathrm{Na}$ ture, 291:61-64.

Delaney, M. L., Bé, A.W.H., and Boyle, E. A., 1985. Li, Sr, Mg, and $\mathrm{Na}$ in foraminiferal calcite shells from laboratory culture, sediment traps, and sediment cores. Geochim. Cosmochim. Acta, 49:13271341.

Delaney, M. L., and Boyle, E. A., 1986. Lithium in foraminiferal shells: implications for high-temperature hydrothermal circulation fluxes and oceanic crustal generation rates. Earth Planet. Sci. Lett., 80: 91-105.

, 1988. Tertiary paleoceanic chemical variability: unintended consequences of simple geochemical models. Paleoceanography, 3: $137-156$.

Elderfield, H., 1986. Strontium isotope stratigraphy. Palaeogeogr., $\mathrm{Pa}$ laeoclimatol., Palaeoecol., 57:71-90.

Elderfield, H., and Gieskes, J. M., 1982. Sr isotopes in interstitial waters of marine sediments from Deep Sea Drilling Project cores. $\mathrm{Na}$ ture, 300:493-497.

Froehlich, P. N., Klinkhammer, G. P., Bender, M. L., Luedtke, N. A., Heath, G. R., Cullen, D., Dauphin, P., Hammond, D., Hartman, B., and Maynard, V., 1979. Early oxidation of organic matter in pelagic sediments of the eastern equatorial Atlantic: suboxic diagenesis. Geochim. Cosmochim. Acta, 43:1075-1090.

Graham, D. W., Bender, M. L., Williams, D. F., and Keigwin, L. D., 1982. Strontium-calcium ratios in Cenozoic planktonic foraminifera. Geochim. Cosmochim. Acta, 46:1281-1292.

Haq, B. U., Hardenbol, J., and Vail, P. R., 1987. Chronology of fluctuating sea levels since the Triassic. Science, 235:1156-1167.

Katz, A., Sass, E., Starinsky, A., and Holland, H. D., 1972. Strontium behavior in the aragonite-calcite transformation: an experimental study at $40^{\circ}-98^{\circ}$. Geochim. Cosmochim. Acta, 36:481-496.

Kinsman, D. J., 1969. Interpretation of $\mathrm{Sr}^{2+}$ concentrations in carbonate minerals and rocks. J. Sediment. Petrol., 39:486-508.

Lea, D., and Boyle, E. A., 1989. Barium content of benthic foraminifera controlled by bottom-water composition. Nature, 338:751-753.

Miller, K. G., and Fairbanks, R. G., 1985. Oligocene to Miocene carbon isotope cycles and abyssal circulation changes. In Sundquist, E. T., and Broecker, W. S. (Eds.), The Carbon Cycle and Atmospheric
$\mathrm{CO}_{2}$ : Natural Variations Archean to Present. Am. Geophys. Union Monogr., 32:469-486.

O'Neil, J. R., Clayton, R. N., and Mayeda, T. K., 1969. Oxygen isotope fractionation in divalent metal carbonates. J. Chem. Phys., 51: 5547-5558.

Palmer, M. R., and Edmond, J. M., 1989. The strontium isotope budget of the modern ocean. Earth Planet. Sci. Lett., 92:11-26.

Pitman, W. C., 1978. Relationship between eustacy and stratigraphic sequences of passive margins. Geol. Soc. Am. Bull., 89:1389-1403.

Renard, M., 1986. Pelagic carbonate chemostratigraphy $\left(\mathrm{Sr}, \mathrm{Mg},{ }^{18} \mathrm{O}\right.$, $\left.{ }^{13} \mathrm{C}\right)$. Mar. Micropaleontol., 10:117-164.

Richter, F. M., and DePaolo, D. J., 1988. Diagenesis and $\mathrm{Sr}$ isotope evolution of seawater using data from DSDP 590B and 575. Earth Planet. Sci. Lett., 90:382-394.

Savin, S. M., 1977. The history of the Earth's surface temperature during the past 100 million years. Ann. Rev. Earth Planet. Sci., 5: 319-355.

Sawlan, J. J., and Murray, J. W., 1983. Trace metal remobilization in the interstitial waters of red clay and hemipelagic marine sediments. Earth Planet. Sci. Lett., 64:213-230.

Shackleton, N. J., 1987. The carbon isotope record of the Cenozoic: history of organic carbon burial and of oxygen in the ocean and atmosphere. In Brooks, J., and Fleet, A. J. (Eds.), Marine Petroleum Source Rocks: London (Blackwell Sci. Publ.). Geol. Soc. Spec. Publ., 26:423-434.

Shackleton, N. J., and Hall, M. A., 1984. Carbon isotope data from Leg 74 sediments. In Moore, T. C., Jr., Rabinowitz, P. D., et al., Init. Repts. DSDP, 74: Washington (U.S. Govt. Printing Office), 613-619.

Shipboard Scientific Party, 1988a. Explanatory notes. In Backman, J., Duncan, R. A., et al., Proc. ODP, Init. Repts., 115: College Station, TX (Ocean Drilling Program), 17-42.

Shipboard Scientific Party, 1988b. Site 709. In Backman, J., Duncan, R. A., et al., Proc. ODP, Init. Repts., 115: College Station, TX (Ocean Drilling Program), 459-588.

Vincent, E.. and Berger, W. H., 1985. Carbon dioxide and polar cooling in the Miocene: the Monterey Hypothesis. In Sundquist, E. T., and Broecker, W. S. (Eds.), The Carbon Cycle and Atmospheric $\mathrm{CO}_{2}$ : Natural Variations Archean to Present. Am. Geophys. Union Monogr., 32:455-486.

Date of initial receipt: 12 January 1989

Date of acceptance: 5 January 1990

Ms 115B-178 